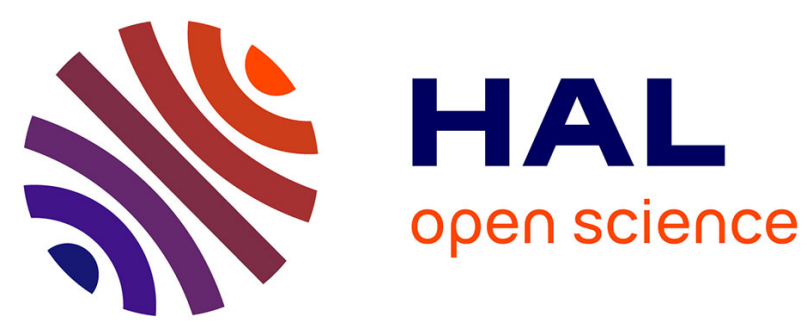

\title{
Connectivity and Automation as Enablers for Energy-Efficient Driving and Road Traffic Management
}

Bassel Othman, Giovanni de Nunzio, Antonio Sciarretta, Domenico Di

Domenico, Carlos Canudas de Wit

\section{- To cite this version:}

Bassel Othman, Giovanni de Nunzio, Antonio Sciarretta, Domenico Di Domenico, Carlos Canudas de Wit. Connectivity and Automation as Enablers for Energy-Efficient Driving and Road Traffic Management. Lackner M., Sajjadi B., Chen WY. (eds). Handbook of Climate Change Mitigation and Adaptation, Springer, pp.XXVII, 2130, 2021, 978-1-4614-6431-0. 10.1007/978-1-4614-6431-0_128-1 . hal-03161894

\section{HAL Id: hal-03161894 \\ https://hal.science/hal-03161894}

Submitted on 8 Mar 2021

HAL is a multi-disciplinary open access archive for the deposit and dissemination of scientific research documents, whether they are published or not. The documents may come from teaching and research institutions in France or abroad, or from public or private research centers.
L'archive ouverte pluridisciplinaire HAL, est destinée au dépôt et à la diffusion de documents scientifiques de niveau recherche, publiés ou non, émanant des établissements d'enseignement et de recherche français ou étrangers, des laboratoires publics ou privés. 


\title{
Connectivity and automation as enablers for energy-efficient driving and road traffic management
}

\author{
Bassel Othman ${ }^{1,2, \star}$, Giovanni De Nunzio ${ }^{1}$, Antonio Sciarretta ${ }^{1}$, \\ Domenico Di Domenico ${ }^{1}$, Carlos Canudas-de-Wit ${ }^{2}$
}

\begin{abstract}
In the member countries of the Organization for Economic Co-operation and Development (OECD), projections show that the improved energy efficiency in transportation may lead to a net decline of about $2 \%$ in energy use until 2040, thus outpacing the predicted increase of vehicle-miles traveled (VMT). However, in OECD-Europe, transportation still represents the biggest source of carbon emissions, contributing by about $25 \%$ to the total $\mathrm{CO} 2$ emissions, with cars and vans representing more than two thirds of this share. The situation is even more alarming in non-OECD countries, where the transportation energy demand is expected to rise by $64 \%$ until 2040 . The shift that we are witnessing toward the adoption of connected and automated vehicles (CAVs) is going to be perhaps, the most disruptive since the early days of automobiles and could revolutionize movement of people and goods. This level of connectivity and autonomy will transform transportation in several dimensions with important societal and economic impacts: improved safety, increased comfort, time saving potential, and more efficient road utilization are among the most widely discussed positive impacts of CAVs. However, the potential energy efficiency benefits of these technologies remain uncertain. From a single-vehicle efficiency perspective, research suggests that lightweight, low-speed, autonomous vehicles have the potential to achieve fuel economy an order of magnitude higher than current cars. Yet, at system-wide level, current estimates suggest that the total energy consumption impacts can range from a $90 \%$ decrease to a $200 \%$ increase in fuel consumption as compared to a projected 2050 baseline energy consumption. The paradigm that traffic congestion mitigation should reduce CO2 emissions is yet to be proved. Therefore, interest in transportation regulation problems with explicit environmental considerations is growing. This work takes a more in-depth look at increased opportunities for energy-efficient driving with energy-oriented traffic management and CAVs deployment. In particular, the focus will be put on the road traffic control strategies in urban networks using connectivity to enable variable speed limits and traffic light adaptive control, as well as the energy-saving opportunities that arise for individual CAVs by anticipating future road geometry, traffic conditions, and interactions with neighboring vehicles.
\end{abstract}

Keywords - Connected and automated vehicles, Energy efficiency, Traffic management, Cooperative control, Infrastructure control, Optimization, Vehicle Platooning, Mixed traffic

\section{Introduction}

Today, transport is one of the most energy-intensive sectors. In fact, as indicated by IEA (2017b), and represented in Fig. 1, the aggregated data of sixteen countries (Australia, Belgium, Canada, Czech Republic, Finland, France, Germany, Hungary, Italy, Japan, Korea, Luxembourg, New Zealand, Spain, the United Kingdom and the United States) reveal that it accounted for the highest share of worldwide energy consumption in 2017 (36\%). For example, passenger cars alone used more energy than the whole residential sector. According to U.S. Energy Information Administration (2017), the transport sector accounted for $55 \%$ of the total liquid fuels consumption, and its share is not expected to decrease for the next two decades because of the increasing travel demand.

The detailed breakdown of energy consumption in the transport sector can be found in IEA (2017a) for the same sixteen countries in 2017, and is represented in Fig. 2. The international aviation, marine bunkers and pipeline transport are excluded. It appears that the transport sector is largely dominated by road vehicles $(88 \%)$, especially passenger cars and freight road, that account for $86 \%$ of the global energy consumption. This highlights the potential of cars and trucks control to reduce energy consumption and greenhouse gas emissions.

The current development of connected and automated vehicles (CAVs) constitutes beyond dispute a significant breakthrough in the movement of people and goods (Sciarretta \& Vahidi, 2020a). On the one hand, the number

\footnotetext{
* Corresponding author (bassel.othman@ifpen.fr)

${ }^{1}$ IFP Energies nouvelles, Rond-point de l'échangeur de Solaize, BP 3, Solaize 69360, France

${ }^{2}$ University Grenoble Alpes, CNRS, Inria, Grenoble INP, GIPSA-lab, Grenoble 38000, France
} 


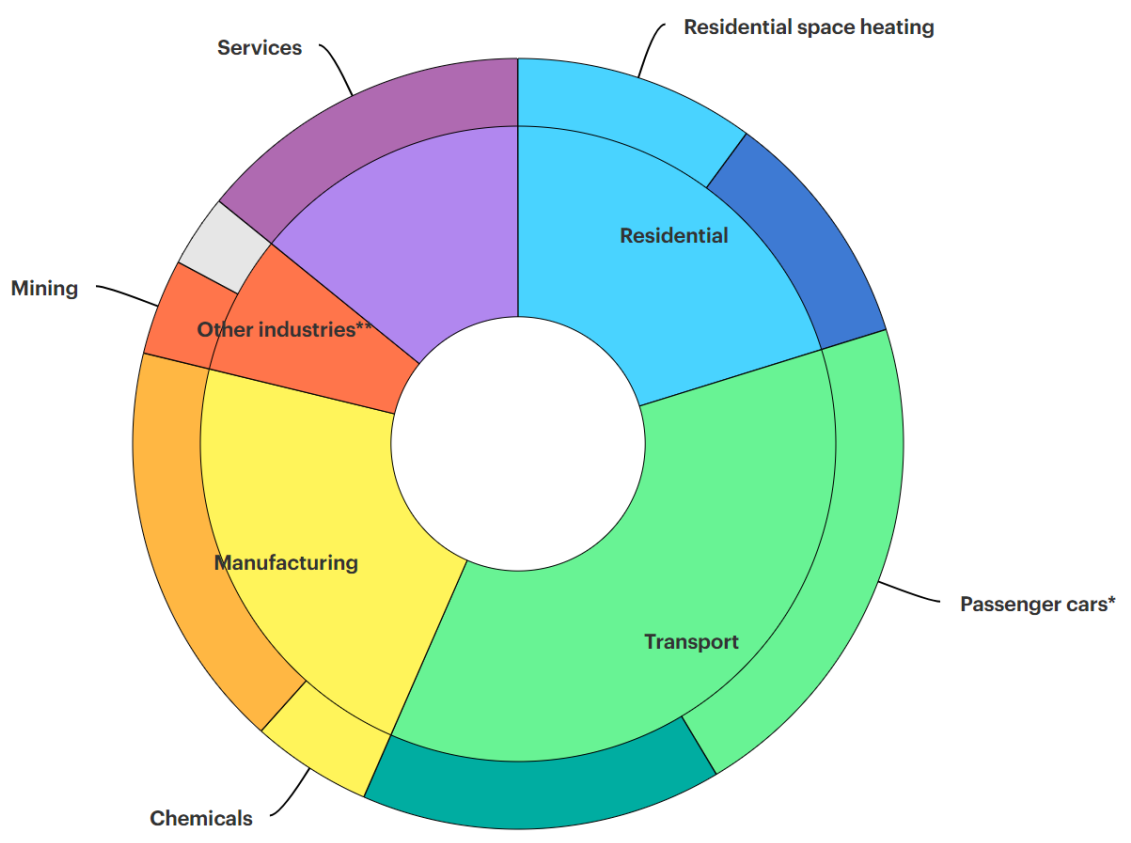

* Passenger cars includes cars, sport utility vehicles and personal trucks.

** Other industries includes agriculture, mining and construction.

Figure 1: Largest end-uses of energy by sector in selected International Energy Agency (IEA) countries (IEA, 2017b) [C2017 IEA. All rights reserved].

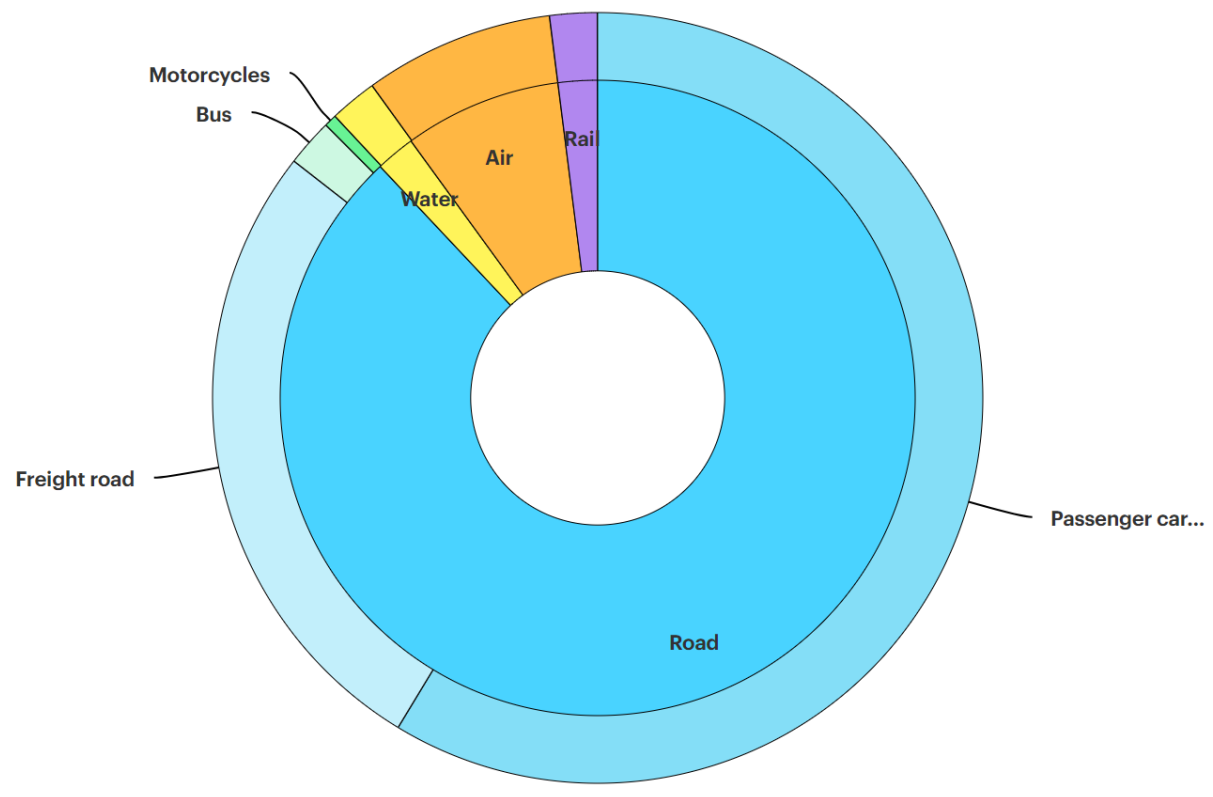

* Passenger cars includes cars, sport utility vehicles and personal trucks.

Figure 2: Energy consumption in transport in selected International Energy Agency (IEA) countries (IEA, 2017a) [C2017 IEA. All rights reserved].

of connected vehicles, i.e. that are able to communicate bidirectionally with other systems, is increasing. Two kinds of connectivity are usually distinguished: Vehicle-to-Infrastructure (V2I)/Infrastructure-to-Vehicle (I2V) communication and Vehicle-to-Vehicle (V2V) connectivity. On the other hand, research and development of fully 
automated vehicles, i.e. that are capable of moving safely without human input, are promising. According to Litman (2017), they are expected to represent 20-40\% of the sales by 2030 .

Thanks to their connectivity features, CAVs are capable of processing and sharing a large amount of data from many sources. Also, their full autonomy ensures much shorter reaction times and more precise positioning and control than conventional vehicles. This results in high reliability and ability to anticipate which offer huge potentials for improving safety and comfort, and reducing travel time and fuel consumption. These opportunities are increased through higher CAVs penetration rates as cooperative control possibilities (vehicles acting cooperatively) are enhanced.

But at a broad level, it is not yet clear whether a high penetration rate of CAVs will have a positive or a negative impact on fuel consumption and CO2 emissions. In fact, Wadud et al. (2016) explain that some effects of the advent of CAVs will reduce energy consumption (development of platooning and eco-driving, congestion mitigation, vehicle light-weighting and right-sizing, de-emphasized performance, car-sharing, on-demand mobility, and reduced infrastructure footprint), whereas other effects will increase it (travel cost reduction, higher highway speeds, increased vehicle features, and new user groups). According to Brown et al. (2014), the impact of CAVs can range from a $90 \%$ decrease to a $200 \%$ increase in global fuel consumption as compared to a projected 2050 baseline scenario. The outcomes depend on the direction that will be favored by future policy making.

As explained previously, CAVs technology is largely based on the exchange of different types of data between vehicles $(\mathrm{V} 2 \mathrm{~V})$ and with the infrastructure $(\mathrm{V} 2 \mathrm{I} / \mathrm{I} 2 \mathrm{~V})$. Two data types may be differentiated:

- Geographic data (time-invariant) that can be embedded on-board or can be accessible via a Geographic Information System (GIS) server: road network architecture, road elevation and slope, road speed limits, speed bumps, road narrowings, safe speeds on curved roads, etc.

- Traffic data (time-variant) that are provided by other vehicles or by the infrastructure (through sensors, cameras, etc.): traffic information, road closings, state of traffic light signals, weather-related road conditions, etc. Note that in absence of real-time data, historical data can be used to predict traffic slow-downs by systematic evaluation (Wan et al., 2017).

In order to predict traffic speed evolution from the available data, CAVs use traffic simulation models that can be deterministic, probabilistic, or based on machine learning approaches (Asadi et al., 2010). With this knowledge, CAVs behave in order to optimize a criterion, e.g., energy consumption. In practice, CAVs recalculate their most energy-efficient routes, and optimize their future speed profiles in anticipation of surrounding vehicles motion, road slopes and geometry, traffic light signals, etc. To minimize their energy consumption, CAVs can also use fuel cut-off, cylinder deactivation, thermal load management, and power split in hybrid electric vehicles (HEVs) powertrains. Connectivity and automation not only enable vehicles to minimize the energy consumption of each vehicle individually ("individual gain" optimization), but also opens the way for vehicles cooperative control ("common good" optimization). Note that such control frameworks could be implemented for conventionally driven vehicles, but they are much more efficient for CAVs because of the certainty of predictions and the precision of powertrain control.

A reliable energy consumption model (fuel and/or electricity) is necessary for CAVs to optimize their future behavior (choice of route and speed profile) in an energy-efficient way. A first possibility is to use a physical model. As described in detail by Sciarretta and Vahidi (2020b), the inputs of such a model should include the road grade angle and the vehicle's parameters and longitudinal dynamics. This latter is governed by Newton's second law of motion, described as

$$
m \frac{d v(t)}{d t}=F_{\mathrm{p}}(t)-F_{\mathrm{res}}(t)-F_{\mathrm{b}}(t)
$$

where $m$ and $v(t)$ are respectively the vehicle's mass and speed. The forces $F_{\mathrm{p}}(t), F_{\mathrm{res}}(t)$, and $F_{\mathrm{b}}(t)$ denote respectively the powertrain forces, the road load, and the force applied by frictional brakes. Assuming a straight road with no wind, the vehicle's energy requirement at the wheels $E_{\mathrm{W}}$ can be expressed as

$$
E_{\mathrm{W}}=\frac{1}{2} m\left(v_{\mathrm{f}}^{2}-v_{\mathrm{i}}^{2}\right)+m g\left(\Delta z+C_{\mathrm{rr}} \Delta s\right)+\frac{1}{2} \rho_{\mathrm{a}} A_{\mathrm{f}} C_{\mathrm{D}} \int_{0}^{t_{\mathrm{f}}} v^{3}(t) d t
$$

where $v_{\mathrm{i}}$ and $v_{\mathrm{f}}$ denote the vehicle's initial and final speeds (at time 0 and $t_{\mathrm{f}}$ ). The variables $\Delta z$ and $\Delta s$ are respectively the total elevation change and the horizontal distance covered during the trip. The parameters $g, C_{\mathrm{rr}}, C_{\mathrm{D}}, A_{\mathrm{f}}$, and $\rho_{\mathrm{a}}$ denote respectively the gravitational constant, the coefficient of rolling resistance, the aerodynamic drag coefficient, the vehicle's frontal area, and air density.

To go further, the energy required from the powertrain, denoted $E$, takes into account the energy dissipated in friction brakes that are acting on the wheels. The energy $E$ can calculated as follows

$$
E=E_{\mathrm{W}}+\int_{0}^{t_{\mathrm{f}}}\left(1-k_{\mathrm{b}}\right) F_{\mathrm{b}}(t) v(t) d t
$$

Usually, the frictional brake force $F_{\mathrm{b}}$ can only be positive when the powertrain force $F_{\mathrm{p}}$ is equal to zero. The parameter $k_{\mathrm{b}}$ denotes the ratio of regenerative braking, i.e. the energy recuperated by the powertrain and stored in the onboard tank. An ideal vehicle with a perfect recuperation $\left(k_{\mathrm{b}}=1\right)$ would result in no energy dissipated 
and $E=E_{\mathrm{W}}$. The complete methodology to determine the expression of $E$ can be found in Sciarretta and Vahidi (2020b).

Kubička et al. (2016) propose to use this kind of model to predict a vehicle's energy consumption for control purposes. Similarly, De Nunzio and Thibault (2017) use an energy consumption model to predict the driving range of electric vehicles (EVs), under different hypotheses. Note that the modeling and the control approaches are much more complex when considering EVs and HEVs because of their limited range, recharge times, and ability to regenerate energy during deceleration phases.

Based on Eq. 2, it appears that there are two ways for a vehicle to reduce its wheel energy, namely

- minimize the distance traveled $\Delta s$, which is fairly trivial;

- minimize the last term, i.e. the energy lost to aerodynamic drag, as it is the only one that is influenced by the vehicle's speed profile during the trip.

Instead of using a physical model, another possibility is to use a data-driven model (S. Zegeye et al., 2013; Qi et al., 2004; Z. Xu et al., 2017; Othman et al., 2020). Basically, it consists in measuring fuel consumption or CO2 emissions and deducing look-up tables, regression models, or machine learning approaches to estimate the energy consumption from the vehicle dynamics. The measurements can be made indoors using a chassis dynamometer, or outdoors during on-road experiments using a portable emissions measurement system (PEMS) (Y. Huang et al., 2018).

It is generally acknowledged that the impact of vehicular mobility on climate change, i.e. the amount of CO2 emitted by vehicles, is proportional to the fuel consumption. The reason is that the relationship between fuel consumption and CO2 emissions is almost linear (Pinto \& Oliver-Hoyo, 2008).

In real driving conditions, it is essential to bear in mind that several factors contribute to an increase in energy consumption compared to the certification value. Fontaras et al. (2017) indicate that these factors include, inter alia, certification margins, vehicle's mass and rolling resistance, wind and temperature conditions, additional electric auxiliaries, air conditioning, road grade, and traffic conditions. The impact of these factors are quantified in Table 1. According to Fontaras et al. (2017), the actual fuel consumption could be increased, on average, by $24 \%$ to $54 \%$ in comparison with the official value.

Table 1: Impact of factors contributing to an increase in energy consumption compared to the certification value, according to Fontaras et al. (2017).

\begin{tabular}{|c|c|c|c|}
\hline Factor & Description & Impacts & Global impact \\
\hline Certification margins & $\begin{array}{c}\text { Vehicle adjustment } \\
\text { Homologation margin } \\
\text { Protocol design \& boundaries }\end{array}$ & $\begin{array}{l}+6 \% \\
+5 \% \\
+4 \%\end{array}$ & $+15 \%$ \\
\hline Mass & $100 \mathrm{~kg}$ extra mass & & $+3.2 \%$ \\
\hline Aerodynamics & $\begin{array}{c}\text { Real world aerodynamics } \\
\text { \& side winds }\end{array}$ & & $+2.2 \%$ \\
\hline Rolling resistance & $\begin{array}{c}\text { Lower energy class } \\
\text { Maintenance } \\
\text { Winter tyre } \\
\text { Wet road }\end{array}$ & $\begin{array}{l}+1.7 \% \\
+0.2 \% \\
+0.2 \% \\
+0.3 \%\end{array}$ & $+2.4 \%$ \\
\hline Temperature & Annual temperature variation & & $+3.5 \%$ \\
\hline Road grade & $0.25 \%$ average grade & & $+2.5 \%$ \\
\hline Additional electric auxiliaries & Air conditioning excluded & & $+5 \%$ \\
\hline Air conditioning & & & $+5 \%$ \\
\hline Traffic conditions & Congestion level \& traffic demand & & $\pm 15 \%$ \\
\hline Total & & & $\begin{array}{c}\text { Between }+23.8 \% \\
\text { and }+53.8 \%\end{array}$ \\
\hline
\end{tabular}


This chapter provides a general review of Cooperative, Connected and Automated Mobility (CCAM) control approaches for improved energy efficiency of road traffic management. The body of the chapter is organized as follows. Section 1 presents the control frameworks of individual CAVs, i.e. the "individual gain" optimization approaches. In Section 2, the main cooperative vehicle control strategies, i.e. the "common good" optimization approaches, are reviewed. Section 3 introduces road infrastructure control methods. Finally, a summary and some future research directions are proposed at the end of the chapter.

\section{Connected Vehicle Control}

The anticipation and prediction potential of connected vehicles opens the door to much more energy-efficient control approaches. CAVs are even more promising because of their more precise positioning and control. In this section, we present the control of vehicles aiming at improving their own energy efficiency, without consideration of the neighboring vehicles energy consumption ("individual gain" optimization).

Two main strategies can be distinguished to improve the energy efficiency of connected vehicles: eco-routing that consists in route choice optimization, and eco-driving that corresponds to the optimization of vehicles speed profiles. They are presented in the following, and should be used jointly to increase energy efficiency (Thibault et al., 2018). For the sake of completeness, note that an energy-efficient driving would also include strategic decisions such as vehicle purchase, maintenance, reduction of on-board elements weight, and reduction of the use of air conditioning systems. For example, Lee et al. (2013) indicate that during idling condition, the fuel consumption of a conventional gasoline engine vehicle could increase up to $90 \%$ because of air-conditioning operation. An overview of the techniques and algorithms to improve energy efficiency of CAVs is proposed by Guanetti et al. (2018).

\section{$1.1 \quad$ Eco-routing}

Route choice is a major factor in vehicles energy consumption. Intuitively, a driver would usually choose the shortest travel distance or the fastest travel time route. However, these are not always the most energy-efficient routes because the shortest route may be partially congested, and the fastest route may be longer and contain high speed limit segments (Y. Huang et al., 2018). Kuo (2010) proposes a model that indicates that the most energy-efficient route could have $25 \%$ reduction in fuel consumption over the fastest route and $23 \%$ over the shortest route. As a result, a Swedish study presented by Ericsson et al. (2006) indicates that in $46 \%$ of cases the drivers' spontaneous route choices were not the most fuel-efficient routes.

Hence, an energy-efficient driving should integrate an eco-routing algorithm that optimizes the route choice in terms of energy consumption. The eco-routing optimization problem can be formulated as follows

$$
\begin{array}{cl}
p^{*}(o, d)=\underset{p \in \mathcal{P}}{\operatorname{argmin}} \sum_{k \in p} g_{k}\left(\theta_{k}\right) \\
\text { subject to } \begin{array}{c}
n_{0}=o, \\
n_{|p|}=d .
\end{array}
\end{array}
$$

where $\mathcal{P}$ is the set of all paths $p=\left\{n_{0}, \ldots, n_{d}\right\}$ between origin $o$ and destination $d$, and $p^{*}$ is the optimal route. Function $g_{k}$ returns the energy consumption associated with the travel on segment $k$. Usually, $g_{k}$ depends on the parameters of segment $k$, that can be time-dependent and are denoted $\theta_{k}$ (road congestion, road type and grade, speed limit, traffic light signals, etc.). These parameters have a great impact on energy consumption. For example, Jin et al. (2016) indicate that a vehicle traveling on a 250 -meter freeway section with a $6 \%$ grade increases its fuel consumption of $86 \%$ in comparison with the same section, with identical initial speed, final speed, and trip time, but with a $0 \%$ grade.

In addition of energy consumption, energy-efficient control strategies also have to pay special attention to travel time and distance. Hence, some eco-routing algorithms formulate the optimization problem with the travel time explicitly appearing either in the constraints (Zeng et al., 2016) or in the objective function (De Nunzio et al., 2017a). In the case of a bi-objective function, the solution found can be a Pareto-Optimal route.

Different methods can be used to solve the eco-routing optimization problem. For example, some authors implement Dijkstra-type algorithms (Kluge et al., 2013), while others use heuristic searches (Nannicini et al., 2012). To reduce the set of possible solutions, eco-routing algorithms can consider additional constraints on the maximum travel distance. A comparison of different eco-routing formulations is proposed by Kubička et al. (2016): one of them averages the observed energy consumption, another one estimates fuel consumption with a regression model, and the last one uses a physical energy model. De Nunzio et al. (2017b) present an innovative eco-routing algorithm especially adapted to EVs in an urban environment, and that considers the impact of onboard accessories and systems on the electricity consumption. The approach considers historical map data and available average traffic speeds to optimize the route choice. De Nunzio et al. (2018) propose a formulation adapted to HEVs, in which a constraint on the final SoC is defined. However, the eco-routing optimization is relaxed and solved as a standard shortest-path problem (SPP), the SoC constraints being a-posteriori enforced. 
The optimization is based on a semi-analytical strategy that ensures fast and accurate solutions. Ojeda, Chasse, and Goussault (2017) present an approach related to energy-efficient route choice for heavy-duty vehicles, which is quite different from eco-routing strategies as they can only travel on a limited amount of roads. In this work, the approach consists in a precise reconstruction of the characteristics of the road before departure. This kind of tool can be of great interest for fleet managers as it allows them to precisely quantify the fuel cost of different itineraries.

Anticipating the evolution of traffic is a particularly critical point in the development of eco-routing algorithms. This could be enhanced by implementing cooperative eco-routing approaches, in which the impact on energy efficiency is analyzed at the network level, rather than at the vehicle level only (Guanetti et al., 2018).

\section{$1.2 \quad$ Eco-driving}

In addition of eco-routing algorithms that optimize route choice, it is essential to reduce the occurrences and the intensity of the acceleration phases as they are the ones that consume the most energy. In other words, the optimal speed profile in terms of energy efficiency is a constant speed. However, this instruction is usually impossible to follow in real traffic conditions, especially in an urban environment with traffic light signals, road congestion, pedestrians, cyclists, road grade, different speed limits etc. Hence, the core objective of eco-driving is to predict the traffic evolution and deduce accordingly a speed profile as smooth and energy-efficient as possible along a given route (Y. Huang et al., 2018). For example, Xia et al. (2013) present a study that shows that it can be more energy-efficient to have high acceleration and deceleration to catch green lights, rather than idling at red signals and starting from stops.

A key point of eco-driving is the choice of the targeted cruise speed. The objective is to travel at the speed associated with the lowest energy consumption rate, expressed in L/100 km for internal combustion engine vehicles (ICEVs) and in kWh/100 km for EVs. As indicated by Pulkrabek (2004), the energy consumption rate is indeed not constant, as

- firstly, the rate decreases with the increase of vehicle speed. The reason is that heat losses are reduced at higher engine speed;

- then, the rate starts increasing at high vehicle speed due to increased friction losses.

As a result, the curve representing fuel consumption rate as a function of vehicle speed shows a U-shape. The optimal speed depends on the vehicle and engine sizing, and on the powertrain type (diesel, gasoline, EVs, HEVs). Fig. 3 represents the fuel consumption rate-vehicle speed curve for different passenger cars (Yang et al., 2018).

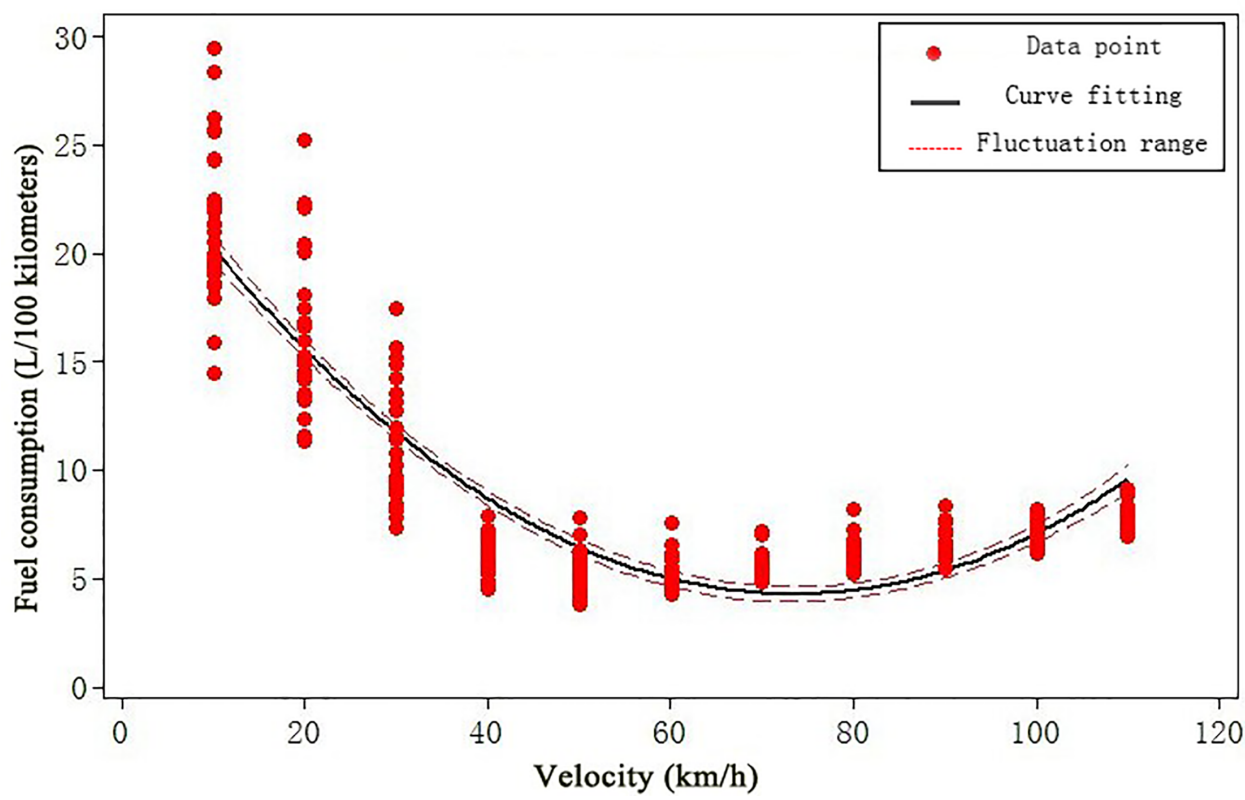

Figure 3: Fuel consumption rate as a function of vehicle speed, based on survey reports of different passenger cars (Yang et al., 2018) [C2018 Yang et al.].

Note that vehicles speed profile optimization impacts many other metrics than energy consumption and CO2 emissions. Hence, eco-driving algorithms may have other complementary objectives: reducing pollutant emissions such as NOx and particulate matter (PM) emissions, increasing safety, increasing passenger's comfort (by ensuring a low jerk), reducing the travel time, etc. 
In the following, a formulation of the eco-driving problem and a discussion about its technical solutions are proposed. Let us denote the state of the vehicle under consideration $x(t)=\{s(t), v(t), \xi(t)\}$, and its control $u(t)=\left\{T_{\mathrm{m}}(t), T_{\mathrm{e}}(t), F_{\mathrm{b}}(t)\right\}$. The variables $s(t), v(t)$, and $\xi(t)$ are respectively the position, the speed, and the state of charge $(\mathrm{SoC})$ of the vehicle at time $t$. The variables $T_{\mathrm{m}}(t), T_{\mathrm{e}}(t)$, and $F_{\mathrm{b}}(t)$ denote respectively the electric motor torque, the internal combustion engine torque, and the forces applied by frictional brakes. This formulation is well adapted to HEVs. Note that for ICEVs, $\xi$ and $T_{\mathrm{m}}$ should not be considered. Similarly, $T_{\mathrm{e}}$ should not be considered with EVs. Based on the vehicle dynamics (cf. Eq. 1), $f$ is the state vector field that returns the new vehicle state from its control and previous state. Function $g$ returns the energy consumption rate from the vehicle state and control. In an urban environment, the eco-driving problem can be separated into subproblems such that for each single segment of the vehicle's route, the following optimization problem is solved

$$
\begin{array}{cl}
\underset{u}{\operatorname{minimize}} & J=\int_{0}^{t_{\mathrm{f}}} g(u, x(t)) d t \\
\text { subject to } & \dot{x}(t)=f(u, x(t)), \\
& 0 \leq s(t) \leq s_{\mathrm{f}}, \\
& v_{\min }(t, s(t)) \leq v(t) \leq v_{\max }(t, s(t)), \\
& \xi_{\min } \leq \xi(t) \leq \xi_{\max }, \\
& s(0)=0, \quad v(0)=v_{\mathrm{i}}, \quad \xi(0)=\xi_{\mathrm{i}}, \\
& s\left(t_{\mathrm{f}}\right)=s_{\mathrm{f}}, \quad v\left(t_{\mathrm{f}}\right)=v_{\mathrm{f}}, \quad \xi\left(t_{\mathrm{f}}\right)=\xi_{\mathrm{f}} .
\end{array}
$$

Sciarretta et al. (2015) present a complete overview of the eco-driving problem constraints and parameterization. In short, Eq. 5c - 5e are the state constraints. In particular, Eq. 5d represents the fluctuation of traffic speed that can be due to road congestion, speed limits, traffic light signals, etc. Eq. $5 \mathrm{f}$ and Eq. $5 \mathrm{~g}$ correspond respectively to the initial and the terminal constraints of the segment under consideration.

Two paradigms aiming at solving eco-driving problems can be differentiated: offline optimizations that assume that all road and traffic characteristics and constraints are known in advance (or at least estimated with increased awareness thanks to connectivity), and online optimizations that use prediction models to perform real-timecapable control, e.g., model predictive control (MPC) approaches (Sciarretta et al., 2015). In practice, offline solutions are based on a horizon $t_{\mathrm{f}}$ that corresponds to the travel time of the whole trip. Conversely, online solutions are based on a much shorter horizon $t_{\mathrm{f}}$, over which the behavior of the preceding and the surrounding vehicles needs to be predicted.

Sciarretta et al. (2015) present some advancements in online eco-driving approaches. For ICEVs, the authors propose a parametric optimization technique inspired by the analytical solution of a simplified version of the eco-driving optimal control problem. For HEVs, a bi-level algorithm that tries to decouple energy-optimal drive control from hybrid energy-management control is presented.

As indicated previously, eco-driving strategies for EVs and HEVs are promising but they introduce additional challenges because of their limited range, recharge times, and ability to regenerate energy during deceleration phases, which add constraints to the optimization problem. Han et al. (2019) explain that even with full regenerative braking, EVs would still benefit from optimized speed profiles that reduce electromechanical energy conversion losses. Concerning ICEVs, the authors show that pulse and glide operation of the engine (chattering optimal control) represent great energy saving potentials, but can be difficult to implement in practice. Finally, the authors indicate that significant energy savings $(5 \%-30 \%$ in each scenario) can be achieved by optimizing speed profiles over conventional driving strategies. In particular, energy savings can result from a better utilization of the energy delivered to the wheels, if vehicles are driven with lower and constant speeds, reducing the aerodynamic drag.

A moot point in the development of eco-driving systems is the necessity to adequately take into account the presence of preceding vehicles. To overcome this issue, Han et al. (2018) present an eco-driving control strategy for electric CAVs. The objective is to optimize the speed profiles of CAVs in order to improve their energy efficiency, while guaranteeing safety constraints, i.e. respecting the minimum inter-vehicle distance and maximum speed limits. The controller is set to solve optimal control problems and return analytical state-constrained solutions under different assumptions. According to the authors, the proposed framework is suitable for online implementations, and significantly improves the energy efficiency without increasing trip time. Similarly, Ojeda, Han, et al. (2017) propose an eco-driving strategy for electric CAVs based on the use of an analytical solution in an MPC framework, which makes it suitable for a real-time use. In this approach, the controller predicts the trajectory of the preceding vehicle under the assumption of constant acceleration. The authors indicate that the algorithm is robust and provides near-optimal speed profiles in terms of energy consumption.

J. Zhu et al. (2019) propose an eco-driving strategy for HEVs that indicates an energy-optimal speed profile to follow. To reduce the computation time and enable online implementation, the authors train two artificial neural networks (ANNs): one of them is used to decide which control sequence to apply, the other one is used to estimate the duration of each control mode in the control sequence. The control sequences include maximal hybrid acceleration, maximal electric acceleration, maximal regenerative braking, maximal hybrid recharge, braking and regenerative braking, optimum hybrid operation, optimum electric operation, and constant speed. These 
multi-class ANNs are trained in order to return optimal speed profiles from the boundary conditions, and the constraints of the upcoming trip, which can be estimated through connectivity and measurements. In practice, the ANNs are trained using a database provided by the offline resolution of optimal control problems through dynamic programming (DP). In fact, DP is an algorithm capable of providing accurate optimal solutions of the problem, but has the disadvantage of a high computational complexity, making real-time implementations of the optimization procedure difficult. The authors indicate that the online ANN-based speed profiles and the offline DP-based speed profiles are reasonably close, both in terms of speed and in terms of energy efficiency. This result highlights the interest of machine learning approaches as they give similar results but have much smaller computation times.

In an urban environment, the broadcast of signal phase and timing (SPaT) through I2V communication represents a real opportunity in terms of energy efficiency. This allows the CAVs to anticipate the future states of traffic light signals (TLS) and optimize their own speed profile for a timely arrival at a green light. This approach, known as Green Light Optimal Speed Advice (GLOSA), can be enhanced by V2V communication as vehicles would also be aware of the traffic state ahead. De Nunzio et al. (2016) propose a strategy in which the speed profile of a vehicle is optimized to improve the energy efficiency by catching the green lights along an arterial road. In this work, the authors consider that the information about several successive signalized intersections is available (I2V), but that vehicles do not communicate with each other (no V2V). As shown in Fig. 4a, several speed trajectories are possible for the vehicle under consideration to cross all the intersections at a green light. The proposed algorithm finds the most energy-efficient possibility among the available ones. The impact on the energy consumption of the penetration rate of vehicles equipped with this technology is given in Fig. 4b. It appears that the higher the penetration rate, the greater the gains in terms of energy efficiency.
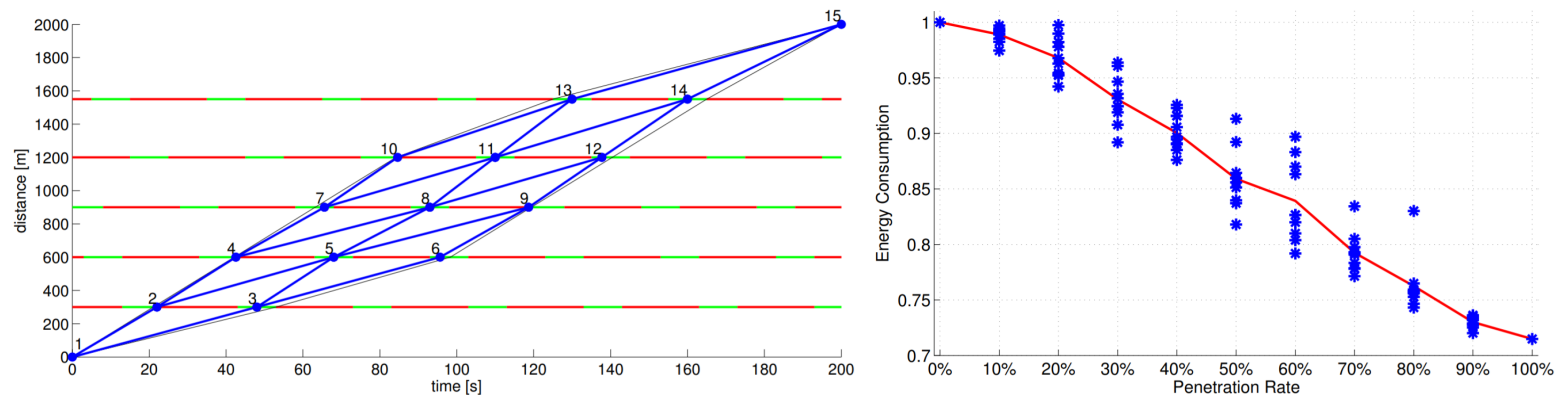

(a) Graph of the possible paths to travel along an arterial (b) Energy consumption as a function of the penroad between nodes 1 and 15 , with 1 node per green phase. etration rate of equipped vehicles.

Figure 4: GLOSA-based eco-driving approach with SPaT broadcast (De Nunzio et al., 2016) [Published with permission of John Wiley and Sons].

To conclude, it is essential to keep in mind that prior knowledge of in-trip and final trip constraints, such as, inter alia, speed limits, road grade and geometry, state of the road, travel time and distance, and final speed, opens the door to greater energy savings thanks to better anticipation (Han et al., 2019). Eco-driving algorithms' efficiency can also be improved with the consideration of other drivers' aggressiveness, which can be easily addressed when considering CAVs, and with the use of more complex models to predict the state of the surrounding vehicles (Ojeda, Han, et al., 2017). Moreover, high CAVs penetration rates offer very good prospects in terms of energy efficiency as they considerably improve the anticipation in CAV driving, car following, and lane selection and merging. A democratization of eco-driving systems would need robust online calculations for all types of powertrains, and the generalization of optimal in-trip advice in all situations, especially in urban areas. This latter is enhanced by the use of $\mathrm{I} 2 \mathrm{~V}$ communication, which represents a great opportunity to develop green waving, and to approach intersections in an energy-efficient way by anticipating SPaT (Sciarretta et al., 2015). According to Guanetti et al. (2018), it is necessary to consider the uncertainty over the bounds on the predicted vehicle speed. Driver safety, performance improvement, and real-time operation are identified as the main technical challenges for the development of connected CAVs control.

\section{Cooperative Vehicle Control}

The first opportunity that is opened up by CAVs consists in the control of vehicles aiming at improving their own energy efficiency, i.e. "individual gain" optimization such as eco-routing and eco-driving approaches presented previously. In this case, CAVs communicate with each other and with the infrastructure, which ensures reliable predictions as the need for guessing is reduced, but vehicles make their decisions individually. As a result, vehicles can appear to be competing in some situations that involve motion conflicts.

Vehicles connectivity and automation offer the possibility to go further and develop vehicles cooperation, i.e. vehicles that communicate $(\mathrm{V} 2 \mathrm{~V}$ and $\mathrm{I} 2 \mathrm{~V})$ and also coordinate their movements. Coordination algorithms 
enable to mitigate conflict situations and guarantee that decisions are jointly feasible (Andersen et al., 2017). Such algorithms also open the door to optimal coordinated control, e.g., minimizing the global energy consumption ("common good" optimization). Vehicles cooperation is almost impossible to implement with conventional human-driven vehicles (HVs) due to unknown plans of neighboring vehicles and lack of precision to coordinate speed, hence the interest in implementing this kind of approach with CAVs (Sciarretta \& Vahidi, 2020a).

In practice, cooperation can consist in vehicle platooning, cooperative adaptive cruise control (CACC), lane changing and merging control, and cooperative intersection control. These points are described and discussed in the following. A key question in vehicles cooperation is the CAVs penetration rate necessary for significant energy efficiency improvement.

\subsection{Vehicle Platooning}

Vehicle platooning consists in the formation of groups of vehicles that travel very closely together. Each platoon is composed of a lead vehicle that controls the speed, and other vehicles that follow the speed setpoint. Vehicle platooning represent a real opportunity in terms of energy efficiency as shorter gaps between the vehicles reduce the aerodynamic drag coefficient. Reduced distances between the vehicles also increase road capacity.

CAVs improve opportunities for vehicle platooning as high speed V2V communication and vehicle automation enable faster reactions resulting in reduced risk of rear-end collision and improved traffic safety. This also allows to reduce the minimum gap between heavy-duty vehicles from about $10 \mathrm{~m}$ (Bonnet \& Fritz, 2000) to $2 \mathrm{~m}$ (Al Alam et al., 2010), ensuring higher energy efficiency and road capacity. For example, Browand et al. (2004) indicate that an 8-10 meters gap results in an average fuel saving of $8 \%$, while a 3-4 meters gap results in an average fuel saving of $11 \%$. Generally speaking, McAuliffe et al. (2017) indicate that the closer the longitudinal spacing, the better the energy efficiency of platoons. For improved energy efficiency, note that the gaps between vehicles could be variable, depending on the road slope (L. Zhang et al., 2020).

According to Bhoopalam et al. (2018), three levels of automated platooning can be defined, as follows

1. human-driven platooning with in-platoon resting in which the lead vehicle is handled by a driver and the following vehicles can complete the driving tasks automatically;

2. hybrid platooning in which the following vehicles can be driverless during platooning phases;

3. driverless platooning which involve full autonomous vehicles.

It appears that vehicle platooning is particularly suitable for the movement of heavy duty vehicles, especially on highways as they are traveling at high speed, causing high aerodynamic drag. The opportunity represented by truck platooning in terms of aerodynamic drag reduction is illustrated in Fig. 5. Also, trucks usually have to travel long distances on highways and could easily join neighboring trucks to form platoons, even if they have different origins and destinations. The first studies about truck platooning for fuel saving purposes started in the mid-1990s with the European project Chauffeur I (Bonnet \& Fritz, 2000). A complete overview of fuel economy in truck platooning can be found in L. Zhang et al. (2020).

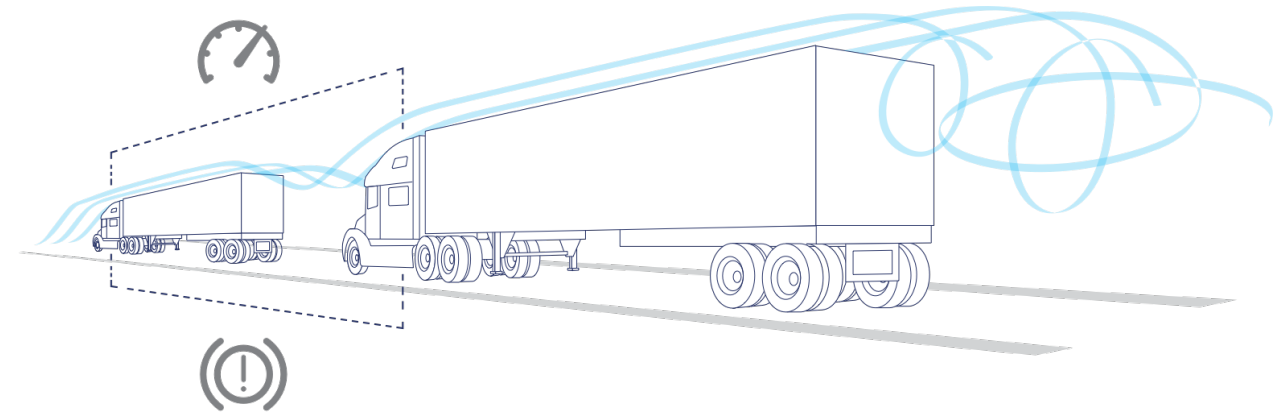

Figure 5: Illustration of the reduction of the aerodynamic drag in truck platooning (Peloton Technology, n.d.) [CPeloton Technology. All rights reserved].

As mentioned above, fuel savings in truck platoons can be maximized through aerodynamic drag coefficient reduction, which is impacted by

- the distance between vehicles, as indicated above;

- the aerodynamic-trailer configuration, e.g., trailer skirts, and trailer boat tails; (L. Zhang et al., 2020).

- the position in the platoon. For example, a study conducted with three Class 8 tractor-trailer trucks indicates that the fuel savings of the lead, the second, and the third trucks were respectively $18 \%, 24 \%$, and 23\% (Lu \& Shladover, 2011). Hence, it seems to be judicious to establish a rotation of the lead vehicle, especially in platoons that are not driverless as it would allow drivers to rest. 
Several methods can be used to analyze the aerodynamic drag coefficient evolution and the potential fuel savings: wind tunnel test, road test, track test, and simulation methods. L. Zhang et al. (2020) discuss the advantages and disadvantages of each method. This kind of approach is useful for determining the impact of wind direction on fuel savings. For example, Marcu and Browand (1999) indicate that there is still an interest in vehicle platooning under crosswind conditions in terms of energy savings.

In practice, the implementation of vehicle platooning poses some issues. Sciarretta and Vahidi (2020a) indicate that the development of truck platooning has resulted in significant challenges, e.g., platoon string stability (avoid the amplification of disturbances from the platoon leader to the downstream vehicles.), communication needs, control design, and formation scheduling. For example, platoons on highways may prevent other vehicles from changing lanes or merging into the highway at on-ramps. This phenomenon is accentuated by longer platoon sizes, especially for high traffic densities. This can be solved by dedicating lanes to CAVs and platooning vehicles (Noorvand et al., 2017), or by developing adapted algorithms based on V2V communication that allow platoons to change lanes, and to yield gaps for merging vehicles (M. Wang et al., 2019). Another key point for realworld development of vehicle platooning is to ensure algorithms of suitable complexity, especially in case of large number of vehicles platooning. Some researchers have worked in this direction. For example, Van De Hoef et al. (2015) propose to formulate the problem of controlling a large number of trucks in a coordinated manner by using clusters. Similarly, Van de Hoef (2016) presents a heuristic approach based on an iterative algorithm. A crucial point in CAVS platooning consists in the communication needs. In fact, an interruption in the communication with a small distance between vehicles can pose serious safety problems in the case of emergency braking of the lead vehicle. Hence, it is safer to keep large gaps ahead of the platoon leader (Shladover et al., 2015).

Today, truck platooning technology has matured and major manufacturers could penetrate the market in the near future (Sciarretta \& Vahidi, 2020a). In 2016, a European project, namely the EU Truck Platooning Challenge, gave rise to the first cross border truck platooning initiative in the world. Six truck platoons (one of each brand: DAF, Daimler, IVECO, MAN, Scania and Volvo) departed from different European cities to arrive in Rotterdam at the same time. Aarts and Feddes (2016) present the main results of the project and the essential points for further development of real-world truck platooning: involvement of the end users (shippers and haulers), clear segmentations showing where platooning can be operational, high definition maps, reliable real-time traffic information, etc.

For improved energy efficiency, vehicle platooning should be combined with complementary strategies, such as the ones presented in Section 1: eco-routing, eco-driving, vehicle choice, maintenance, reduction of weight, reduction of the use of air condition systems, etc. Van De Hoef et al. (2015) propose a centralized approach aiming at forming and controlling truck platoons. The strategy consists in

1. determining the shortest path for each truck according to their origins and destinations;

2. establishing possible platoon configurations;

3. optimizing the speed profiles of each platoon.

\subsection{Cooperative Adaptive Cruise Control}

Similarly, CACC research has been strongly progressing over the past few years. CACC is basically an enhanced adaptive cruise control (ACC) system which, in addition to using range sensors to adjust the distance from the vehicle ahead, anticipates the motion of neighboring vehicles with a much quicker response through $\mathrm{V} 2 \mathrm{~V}$ and/or I2V communication. For example, Milanés et al. (2013) indicate that the communication delay to relay a message from the first to the fourth vehicle can be about only 0.1 seconds with CACC, against more than 5 seconds with autonomous ACC, as they have to witness speed or direction changes of the vehicle in their direct line of sight to react and adapt their own behavior. An illustration of vehicles performing CACC is given in Fig.6.

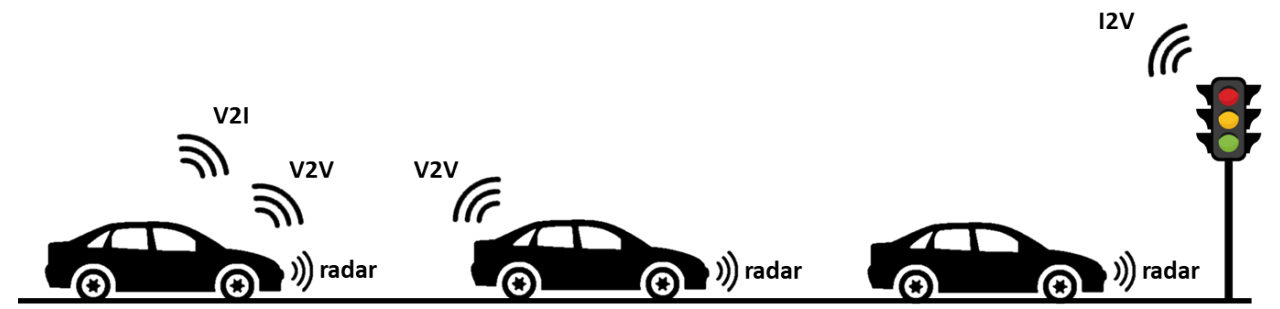

Figure 6: Illustration of vehicles forming a CACC string.

The fundamental difference between vehicle platooning and CACC is that a platoon is necessarily composed of a lead vehicle, that other vehicles are following as precisely as possible. In other words, vehicle platooning consists in a hierarchical control structure, while CACC structures allow communication between all vehicles (Sciarretta \& Vahidi, 2020a). Usually, vehicle platooning is based on a constant-distance gap, i.e. a constant distance that 
does not depend on the speed, while CACC considers a constant-time-gap, i.e. a distance proportional to vehicles speed (Shladover et al., 2015).

The motivations of CACC are very similar to those of vehicle platooning: improving traffic flow, safety, comfort, convenience, customer satisfaction, and decreasing fuel consumption. According to Nowakowski et al. (2010), the use of high-speed communication in CACC systems could reduce the average gap between vehicles from 1.4 seconds to 0.6 seconds, which should result in an increase in energy efficiency and in highway lane capacity. H. Liu et al. (2020) compare the $100 \%$ CACC and the $100 \%$ ACC, and reveal that in the CACC scenario, the fuel consumption rate can be reduced by half, and the capacity can increase up to $49 \%$, depending on the traffic demand. Safety can be improved in CACC systems by developing collision warning or mitigation systems, but CACC alone is not primarily a safety system (Shladover et al., 2015).

CAVs are designed to communicate both with the infrastructure (V2I/I2V), and with other vehicles (V2V). According to Shladover et al. (2015), two major uses of V2I/I2V communication in CACC systems are the implementation of

- variable speed limits (VSL), aiming at reducing congestion at bottleneck locations by lowering upstream vehicle speeds;

- arterial coordinated start, that coordinates the start of vehicles waiting at a red traffic signal when the signal turns green. In urban areas, arterial coordinated start is particularly promising because of its simplicity and potential huge impact on intersections throughput (Lioris et al., 2016).

In CACC systems, V2V communication consists in sharing at least the vehicle location, speed, acceleration, intentions, and performance limitations. This communication can be used simply to develop more performing ACC, but it also opens the door to much more complex CACC systems, in which vehicles are able to anticipate their decisions by communicating with vehicles that are beyond their direct line of sight. This would greatly contribute to stabilizing the responses of the CACC-equipped vehicles. As with vehicle platooning, V2V CACC is especially promising on highways.

In practice, several string formation and dissolution strategies for improved energy efficiency can be implemented, with differing connectivity architectures and collaboration levels. These strategies can be based on, inter alia, classical controllers, receding horizon controllers, constrained optimization based on Pontryagin's Minimum Principle (Dollar et al., 2020a). An overview of these approaches is proposed by Shladover et al. (2015). The simplest approach for string formation consists in ad-hoc clustering, in which vehicles do not deliberately seek out other CACC-equipped vehicles. In this case, the higher the CACC-equipped vehicles penetration rate, the more efficient this approach is as there are statistically more CACC-equipped vehicles following each other. The interest of ad-hoc vehicles clustering is negligible at low market penetration rates.

CACC string formation strategies can also rely on more developed local coordination methods. The idea is to instruct equipped vehicles that are close to each other to speed up or slow down in order to form a cluster. The moot point with this kind of approach is whether or not the benefits of clustering are greater than the losses due to coordination, e.g., acceleration or deceleration and lane changes. This issue is addressed in the COMPANION project led by Scania (Liang et al., 2013). It appears that the amount of fuel saved by a truck catching up a platoon ahead depends on the initial distance to the platoon and the distance to the destination. Hence, as for ad-hoc vehicle clustering, local coordination strategies are more energy-efficient at high penetration rates. The main challenges to local coordination consist in determining the vehicles' positions with a great precision, while considering the traffic conditions, road slopes, etc. This kind of approach gives rise to privacy concerns as they may need to broadcast identifying information about the vehicle to catch up. Finally, it is clear that full autonomous vehicles, i.e. CAVs, represent a geat opportunity for local coordination implementation as they prevent drivers from having to change lanes to join a cluster.

Another vehicle clustering strategy consists in global coordination, i.e. an approach that coordinates vehicles with similar origins and destinations in order to form a cluster before they enter the highway (Larson et al., 2014). Ideally, vehicles' routes and speed profiles are adjusted to arrive at the same time at the highway entry points. This poses a significant challenge because of the uncertainty of road traffic, and requires long-range communication. In practice, it is likely that this kind of approach will make CACC-equipped vehicles wait before enough vehicles have gathered to cluster together and enter the highway. Through this approach, the moot point is the waiting time before departure, which is difficult to get accepted, especially for short trips. Nevertheless, global coordination strategies can be a good alternative at low penetration rates, when ad-hoc clustering and local coordination approaches are inefficient.

CACC string dissolution strategies also require special attention in order to avoid creating new traffic problems. According to Shladover et al. (2015), a vehicle can leave a CACC string by making a lane change without decelerating, or by braking first to deactivate the CACC function. In the future, research on CACC string dissolution approaches needs to be further investigated, especially in unusual cases, such as when multiple consecutive vehicles leave at the same exit. This would require a very high precision, which could be provided by CAVs.

The potential benefits of CACC have already been investigated and quantified through simulations (Van Arem et al., 2006) and real-world experiments (Milanés et al., 2013). In real traffic conditions, Milanés et al. (2013) indicate that $\mathrm{CACC}$ is able to reduce gap variability, and to handle unequipped vehicles cutting in and out. The authors also demonstrate that CACC has a shorter response time and a better string stability than ACC. This 
confirms the potential for a CACC system to attenuate disturbances, and improve highway capacity and traffic flow stability.

Despite the fact that CACC offers interesting prospects in terms of energy efficiency, very little research has been done to explicitly improve the ecological aspect of the problem. Z. Wang et al. (2017) propose an approach aiming at minimizing the platoon-wide energy consumption and pollutant emissions at different stages (sequence determination, gap closing and opening, platoon cruising with gap regulation, and platoon joining and splitting) of the CACC system operation. In comparison with an existing CACC system, the results show that an eco-CACC alternative may reduce the global energy consumption by $1.45 \%$ during the formation of a platoon, and by $2.17 \%$ during platoon joining phases. As indicated previously in Section 2.1 , the benefits of vehicle clustering are much higher (Lu \& Shladover, 2011) in comparison with a scenario in which no cooperative control is implemented.

Note that several incentive strategies can be imagined to develop CACC in the future, e.g., transfer payments from the following vehicles to the lead vehicle as they do not all equally benefit from clustering (Shladover et al., 2015), use of managed lanes with pricing strategies (Qom et al., 2016), etc.

As with vehicle platooning, a limit must be placed on the length of CACC clusters for many reasons, e.g., safety, performance limitations, and integration with conventional vehicles. The main reason for this limit lies in the need to provide sufficient lane-changing gaps for unequipped vehicles. According to Shladover et al. (2015), the range of the wireless $\mathrm{V} 2 \mathrm{~V}$ communication system is not the limiting factor anymore as it reaches more than $300 \mathrm{~m}$.

CACC systems raises the question of vehicles clustering within a string. In fact, the simplest approach is to add vehicles in the rear as they arrive. For safety reasons, the heaviest vehicles should be at the front, especially if there are trucks in the string. However, this complicates the string formation and dissolution problems, and may reduce passengers comfort in light-duty vehicles. Another option is to group vehicles by destination. This would reduce the number of maneuvers during the string formation and dissolution, thereby ensuring smoother speed profiles, improved energy efficiency, and traffic flow (Shladover et al., 2015).

Finally, CACC offers huge opportunities in terms of energy efficiency, throughput, safety, and customer comfort. This can even be enhanced by the development of CAVs, which are fully autonomous. CACC is also compatible with semi-autonomous vehicles, in which the driver needs to control the steering but not the speed of the vehicle, for example. However, this scenario would be less efficient because of human factors such as drivers' reaction time and lack of precision. Drivers would also need a thorough understanding of how CACC works. V2V CACC seems to produce the greatest benefits on highways, while V2I/I2V CACC opens the door to new alternatives in urban environments, especially at intersections. In the future, several challenges regarding CACC string formation and dissolution will have to be met for a large-scale development of CACC. According to Dey et al. (2015), the first step in the deployment of CACC systems is to establish efficient and robust communications under highly dynamic environments. Additional issues would also need to be addressed. These include, inter alia, the legal aspects of crashes caused by failure of the system, users' privacy and security, technology certification, and users' training in the case of vehicles that are not fully autonomous. Guanetti et al. (2018) indicate that future developments of CACC systems should pay special attention to vehicle and string stability with sufficient robustness margins.

\subsection{Lane Change and Merge}

Lane change and merge (into a highway from an on-ramp or exiting to an-off ramp) represent a complex problem, which can be explained by its combinatorial nature and the lack of information about the average speed on the different lanes (Sciarretta \& Vahidi, 2020a). Some examples of lane change and merge maneuvers are shown in Fig. 7. CAVs offer promising prospects for overcoming the difficulties related to this problem by using V2V communication to anticipate the intention of neighboring vehicles and estimate the traffic speed in each lane. The knowledge of the lanes state in advance could greatly improve eco-routing and eco-driving algorithms for CAVs by making much more judicious and smooth lane changes. For example, Kamal et al. (2016) propose a control framework aiming at improving the energy efficiency and the travel time. In this approach, the authors anticipate the behavior of surrounding vehicles to change lane and adjust their speed. Note that in case of lane reductions and moving bottlenecks in congested situations, the speed of the bottleneck and the speed limit play an important role in terms of energy efficiency, traffic flow rate, average speed, and traffic safety (Li \& Sun, 2017).

In addition to the benefits brought to individual vehicles, anticipative lane selection and merging could also have a positive impact at a larger scale, by improving the overall energy efficiency through reduced risks of phantom jam, and smoother velocity trajectories, as indicated by Raboy et al. (2017). These global benefits could be even greater if the vehicles cooperated, i.e. considered the impact of their decisions on their neighboring vehicles, and behaved in a way that optimizes the "common good". In this sense, the vehicles would not act as independent entities, but as elements of a whole whose objective is the overall minimization of energy consumption (Sciarretta \& Vahidi, 2020a). This cooperative lane change and merge problem, with "common good" considerations, can be addressed in two different ways:

- by implementing a collaborative distributed control, in which vehicles optimize their own behavior and share their intentions (Nie et al., 2016; P. Liu et al., 2017); 

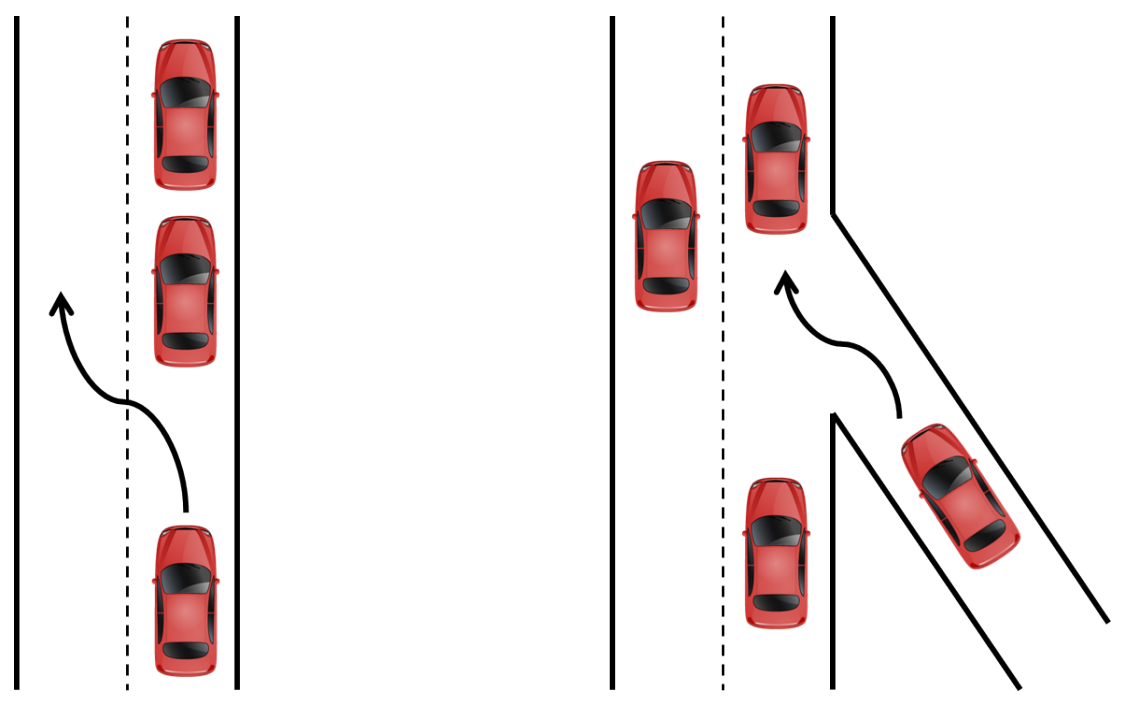

Figure 7: Examples of lane change and merge maneuvers.

- by implementing a centralized control, in which the problem is solved at once for a group of cooperative vehicles.

Dollar et al. (2020b) propose to compare these two approaches in a multi-lane environment. The results indicate that both approaches outperform the classic non-collaborative decentralized controller, i.e. when every vehicle optimizes its behavior to improve its "individual gain". It appears that the centralized algorithm returns the best solutions but its computation time is less suitable for real-time implementation.

In practice, several cooperative lane change and merge strategies can be established. Lombard et al. (2017) propose an approach based on cooperative V2V negotiation. In the same vein, the method presented by Lin et al. (2019) consists in the use of transferable utility games, in which gaps in traffic are created in exchange for monetary compensation. In other words, vehicles can pay to change lane faster and reduce their travel time. The problem is solved using Nash bargaining theory, and the results reveal that this kind of approach can help reach win-win situations.

Only a few studies have evaluated the energy savings of cooperative lane change and merge. One of them is presented by Awal et al. (2015). The authors propose a cooperative strategy that considers vehicles in current and target lanes during lane changing phases. This approach is compared with the traffic model MOBIL, and traffic simulations reveal that the cooperative approach improves merge time and rate, wait time, fuel consumption, average velocity, and flow at the cost of a slightly increased travel time. Similarly, Talavera et al. (2018) propose an extended CACC approach adapted to CAVs, which also ensures lane changes. The authors indicate that the system improves traffic flow, and is also supposed to reduce fuel consumption and CO2 emissions.

Just like for other cooperative vehicle control strategies, the $\mathrm{V} 2 \mathrm{~V}$ communication delay represents a major issue in cooperative lane change and merge approaches. Most of the works on this topic neglect this aspect of the problem. An and Jung (2018) propose a cooperative lane change protocol that integrates the impact of V2V communication delay. The algorithm considers bounding boxes around the vehicles in order to determine the risk of collision. Heß et al. (2018) indicate that experiments through physical test drive and simulation reveal that $\mathrm{V} 2 \mathrm{~V}$ communication systems with $10 \mathrm{~Hz}$ update rate are appropriate.

According to Raboy et al. (2020), efficient cooperative lane change and merge systems should perfectly integrate both vehicle-based radar data and Global Positioning System (GPS) data transmitted through V2V communication. To enhance such systems, vehicles could also use the support of I2V communication. The authors also advocate the implementation of dynamic role assignment during lane change maneuvers, rather than fixed roles established prior to the maneuvers.

\subsection{Cooperative Intersection Control}

In urban areas, signalized intersections have a major impact on the energy consumption of vehicles due to braking, idling, and starting from stops. Currently, many traffic light signal timings are scheduled offline, and are then deployed as fix timetables, that can vary over time, e.g., peak hours/rest of the day, week days/weekend days, etc. To reduce idling and improve throughput at intersections, loop-detectors can be installed, in order to trigger rules to override the pre-optimized timetables, or even to optimize the SPaT of the intersection under consideration online (cf. Section 3.2). For this reason, the states of traffic light signals generally cannot be known in advance with certainty. In such a scenario, it is possible to use historical data in order to estimate the probability of a green or a red over a future horizon (Bodenheimer et al., 2014). 
CAVs offer promising opportunities to improve the energy efficiency at intersections (Sciarretta \& Vahidi, 2020a). In fact, their connectivity and automation features can be used to control them at intersections in a cooperative way, i.e. in a "common good" approach. As explained in Section 2.2, this can be achieved by CACC systems that improve the throughput by allowing coordinated vehicles starts, thanks to I2V communication.

In the near future, it is conceivable that some urban areas may only be accessed by CAVs. In these zones where the penetration rate of CAVs would be $100 \%$, traffic light signals as we know them today would not be needed anymore. For example, Charalampidis and Gillet (2014) propose a framework that optimizes the speed profiles of two vehicles approaching an unsignalized intersection. The study aims at optimizing the travel time and smoothing the CAVs speed profiles for improved passengers' comfort, while ensuring safety constraints. The authors also investigate the effect of the range of the $\mathrm{V} 2 \mathrm{~V}$ communication devices, and indicate that increasing it up to a specific value is beneficial, but the optimal speed profiles do not change after this point. Lin and Jabari (2020) present a cooperative framework that allocates priority at autonomous intersections, based on transferable utility games. In this approach, vehicles can pay for intersection priority. In other words, winners (time buyers) pay losers (time sellers) in each game. Such strategies are promising as they takee into account the value of vehicles' time, and losers are compensated. The authors also indicate that the approach is robust to adversarial behavior. Similarly, Miculescu and Karaman (2019) present an approach based on a polling policy, and Tachet et al. (2016) propose to generalize the classical queuing theory to develop slot-based intersections, similar to those commonly used in aerial traffic. The simulation results reveal that such autonomous intersections could double capacity and significantly reduce delays, compared to conventional intersections ruled by traffic light signals. According to S. Huang et al. (2012), reservation-based intersection control systems could improve the energy efficiency by $50 \%$. In fact, the energy consumption and the number of stops are greatly reduced in such systems (Fayazi et al., 2017; Fayazi \& Vahidi, 2018). Fayazi and Vahidi (2018) indicate that the number of stops can be reduced 100 times, and the improvement in energy efficiency could reach 20\%. Ahmane et al. (2013) propose an illustration, given in Fig. 8, of such an autonomous intersection, in which vehicles negotiate the "right of way" using V2V communication.

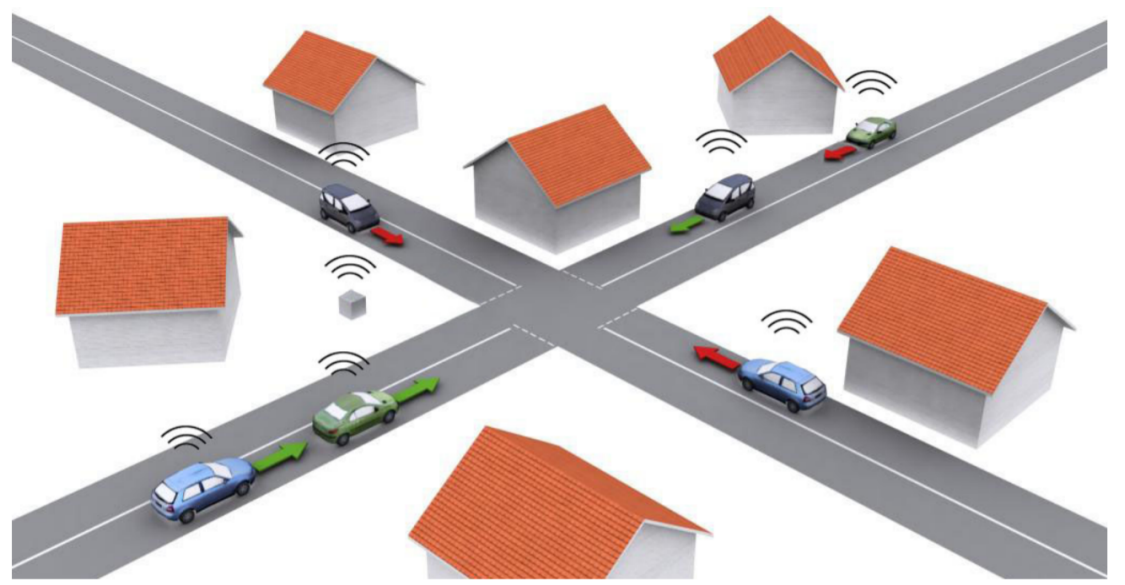

Figure 8: Cooperative intersection (Ahmane et al., 2013) [Published with permission of Elsevier].

More generally, a systematic literature review of intelligent intersection management systems for CAVs is proposed by Namazi et al. (2019). The authors indicate that some works on this topic consider rule-based methodologies (40\%), while others consider optimization methodologies (45\%), and hybrid methodologies (11\%). Currently, few works are based on machine learning approaches (4\%). Namazi et al. (2019) also explain that the vast majority $(93 \%)$ of the articles reviewed consider a CAVs penetration rate of $100 \%$. To be more realistic, autonomous intersections should be compliant with mixed traffic (CAVs and conventional HVs), pedestrians, and cyclists. According to the authors, a promising research direction to achieve this objective is to use the feature of CAVs to collect environmental data and share them through V2V communication. Pedestrians and cyclists could also be spotted by fixed cameras or sensors installed at intersections, and their presence could be indicated to approaching vehicles through I2V communication. To go further, additional analysis and artificial intelligence open the door to pedestrians and cyclists intention prediction (Continental, 2019). Continental is currently working on the development of smarter and safer mobility in cities. An illustration of the company's strategy to anticipate the presence of pedestrians and cyclists is given in Fig. 9.

To go further, intelligent intersection management systems for CAVs can be used in conjunction with CACC systems for increased energy efficiency. Jin et al. (2013) indicate that in high traffic demand scenarios, this kind of approach could shorten the average travel time by $30 \%$ and reduce the fuel consumption by around $23 \%$ with respect to signalized intersections. Note that such approaches slightly sacrifice the energy efficiency to form platoons, which can be challenging in urban areas. 


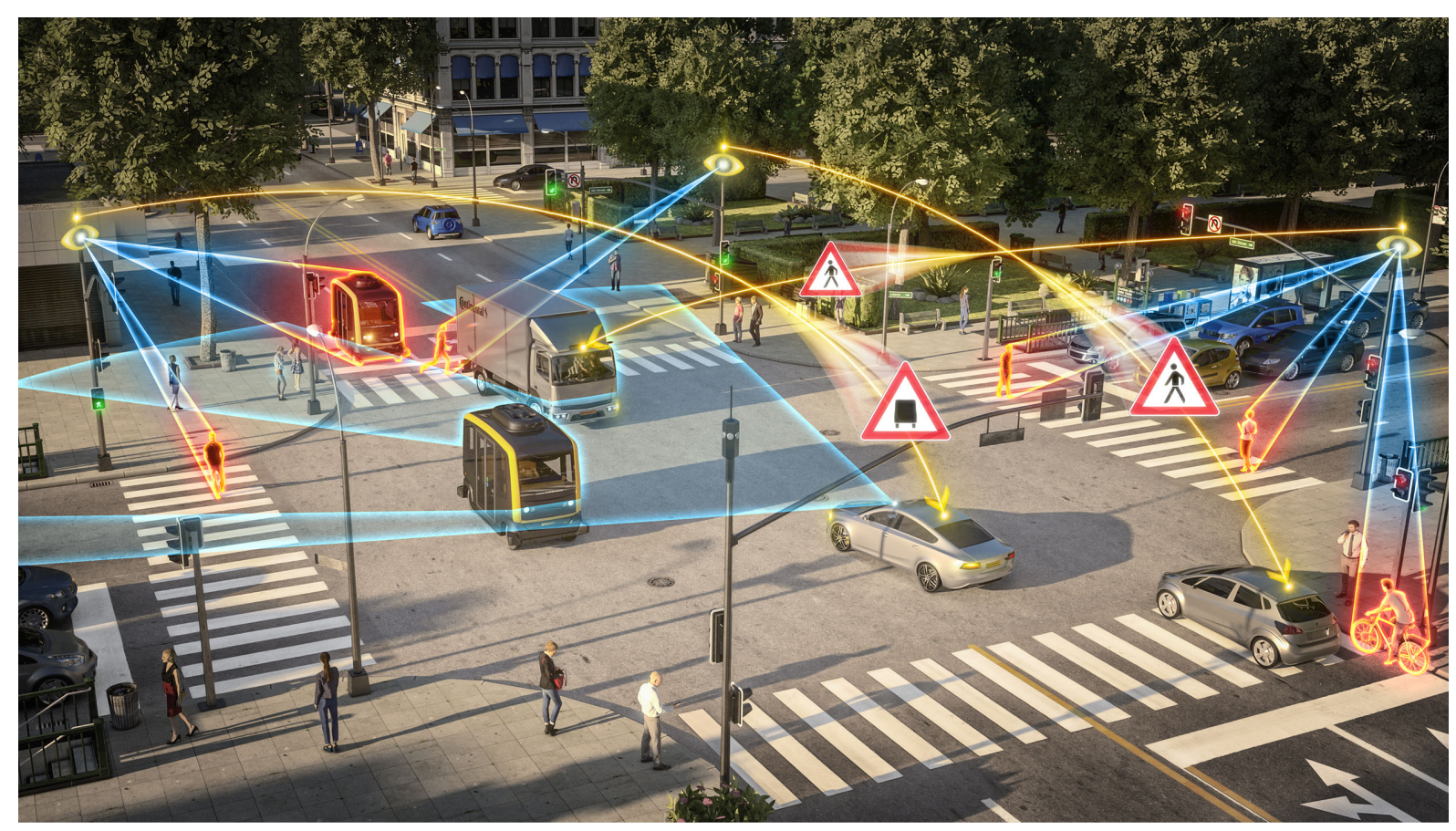

Figure 9: Strategy to increase safety by communicating hidden dangers to approaching vehicles and pedestrians at an intersection (Continental, 2019) [C2019 Continental. All rights reserved].

\subsection{Traffic Harmonization}

CAV technology is a great opportunity to improve traffic fluidity, safety, and energy efficiency. CAVs can optimize their behavior in order to improve their own benefits ("individual gain" optimization), or the global benefits of a group of cooperating CAVs ("common good" optimization).

A key point in CAV research is the management of mixed traffic (CAVs with conventional HVs), as the penetration rate of CAVs will certainly take time before it reaches $100 \%$ (Litman, 2017). In mixed-traffic situations, coordinated and smoother motion of CAVs could also improve the energy efficiency of the surrounding non-automated vehicles, even at low penetration levels (Sciarretta \& Vahidi, 2020a).

In mixed-traffic conditions, HVs have a strong influence on traffic flow as they increase the spread of shock waves in the downstream direction, causing stop-and-go waves (Di Vaio et al., 2019). As indicated by Giammarino et al. (2020), CAVs can be designed to explicitly stabilize the traffic flow and actively dampen stop-and-go waves, which are particularly energy consuming. Vinitsky et al. (2018) present such approaches based on reinforcement learning (RL) strategies. On highways, stop-and-go waves may appear due to the presence of bottlenecks. In this kind of situation, Ghiasi et al. (2019) propose to optimize the speed profiles of the CAVs upstream of the bottleneck in order to hedge against the backward shockwaves and smooth the traffic. According to the authors, the resulting speed harmonization greatly improves the energy efficiency (between $12 \%$ and $16 \%$, depending on the traffic demand) and reduces environmental impacts. Similarly, Liard et al. (2020) present an approach that optimizes the speed profiles of CAVs in order to use them as moving bottlenecks. The authors indicate that this approach is especially beneficial when the density is low and medium, but its impact is limited in highly congested situations. Di Vaio et al. (2019) confirm the robustness and the efficiency of such approaches to dampen down traffic waves, while also considering delays due to V2V communication. Stern et al. (2019) propose to quantify the potential benefits of traffic stabilization by realizing field experiments with 20 to $21 \mathrm{HVs}$ and $1 \mathrm{CAV}$ ( $\sim 5 \%$ of the vehicle fleet) traveling along a circular ring road, as represented in Fig. 10. Vehicle velocity and acceleration data are collected, and emissions are estimated using the MOVES model. The results reveal that the presence of a stabilizing CAV may improve the energy efficiency by 15\%. The potential benefits on pollutant emissions are even greater (up to $73 \%$ reduction for nitrogen oxides). These results are promising, but it is essential to keep in mind that this approach represents a very much tamed scenario, and that less significant impacts are expected when the density of vehicle is lower, or when there are several lanes, as the stop-and-go waves would be rarer and of lower intensity.

The effectiveness of the harmonizing effect of CAVs varies according to traffic conditions and CAVs penetration rate. Y. Zhang and Cassandras (2018) analyze this phenomenon in an urban environment, under mixed-traffic conditions. The authors propose a control strategy for vehicles crossing signal-free intersections, described as follows:

- speed profiles are optimized for the CAVs in order to cross the merging zone in a limited time while 


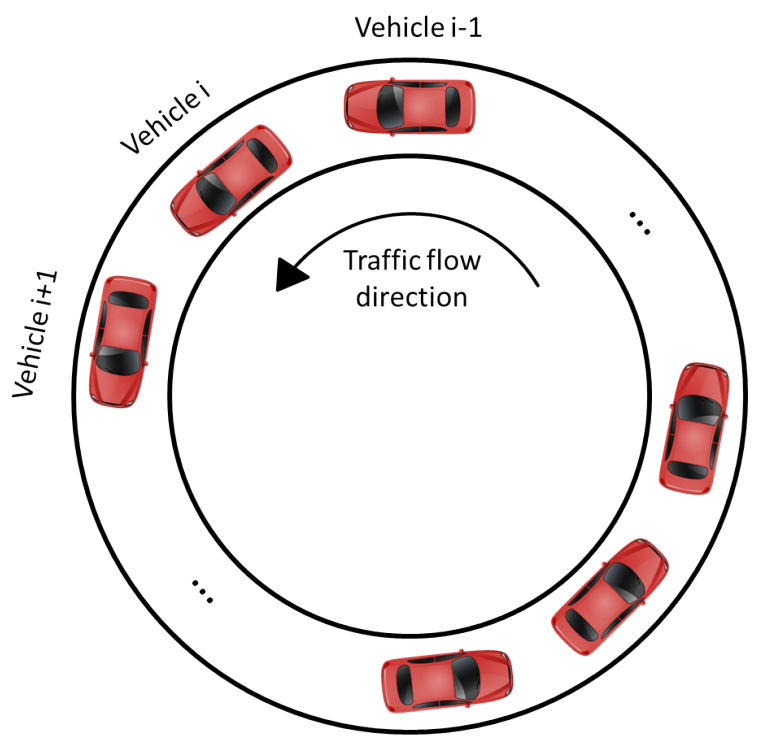

Figure 10: Vehicles traveling along a circular ring road.

minimizing the acceleration and comply with safety requirements;

- HVs are subject to priority rules.

The average fuel consumption is measured for different CAVs penetration rates, and different traffic conditions, and is compared with the energy consumption under TLS control, i.e. conventional signalized intersections. In the scenario proposed by the authors, the interest of CAVs in terms of energy efficiency appears to be obvious because the energy consumption decreases as the penetration rate increases. However, the strategy seems to lose its usefulness under heavy congestion. The authors indicate that the limit below which the approach is beneficial is the critical flow rate, which depends on the road capacity, the speed limit, the number of lanes, etc.

To go further, CAVs cooperation too can have a positive effect on traffic-flow performance and energy efficiency. For example, Van Arem et al. (2006) study the impact of CACC for a highway-merging scenario from four to three lanes. The simulation results indicate that CACC can have a positive impact on traffic throughput, and can increase highway capacity near lane drops. This is particularly true in conditions with high-traffic volume and high penetration rate of CAVs performing CACC. The authors indicate that a low penetration rate could even lead to a degradation of traffic-flow performance. H. Liu et al. (2020) analyze the impact of CACC on energy efficiency in a complex multi-lane mixed-traffic stream. As indicated previously, the fuel consumption rate of the $100 \%$ CACC is reduced by $50 \%$ and the capacity could be increased by $49 \%$, compared to the $100 \%$ ACC scenario. At $40 \%$ penetration rate, the capacity can still be increased by $15 \%$ to $19 \%$ when adapted control strategies are implemented. The study presented by H. Liu et al. (2020) was performed at a simple freeway merging area, but it can easily be extended to more complex scenarios such as complete freeway or arterial corridors.

Note that other CAV-based strategies than CACC can also improve the overall energy efficiency under mixedtraffic conditions. For example, Wan et al. (2016) analyze the energetic impact in an urban environment of a speed advisory system that optimizes CAVs' speed limits in order to reduce idling at red lights. It appears that this approach not only improves the energy efficiency of CAVs equipped with this speed advisory system, but also benefits HVs and decreases their fuel consumption, with a compromise in average traffic flow and travel time. The authors indicate that the global fuel consumption decreases with the increment of CAVs penetration rate. The reason is that HVs are more likely to follow a smoother-moving CAV.

In conclusion, CAVs and their many cooperative strategies have a positive impact on the overall energy efficiency under mixed-traffic conditions. Their impact varies according to traffic congestion and CAVs penetration rate. In order to improve their impact on energy efficiency, CAVs could cooperate, and coordinate several strategies such as, inter alia, CACC, cooperative lane change and merge, autonomous intersection, and speed harmonization.

\section{Road Infrastructure Control}

In Sections 1 and 2, the CAVs control strategies, either for the optimization of their "individual gain" or the "common good", are based on V2V and/or I2V communications. In this section, we review the main infrastructure control strategies. Such approaches are based on V2I communication. In practice, they consist in collecting data from vehicles traveling in the road network (vehicle density, traffic flow, average speed, etc.), which is greatly 
simplified with the current development of connected vehicles. Then, the infrastructure actuators are controlled in order to optimize some metrics (global energy consumption, pollutant emissions, traffic throughput, average travel time, etc.). Usually, these actuators are speed limits and traffic light signals, but they can also include for example dynamic routing, which consists in redistributing the traffic demand over the network in a more efficient way by controlling the split ratios at intersections (Othman et al., 2019).

For optimal CAVs control and maximum energy efficiency, the control of infrastructures, which is based on V2I communication, should be coordinated with the control of CAVs, which is based on V2V and I2V communications.

\subsection{Variable Speed Limits}

One main possibility in the control of road infrastructures is variable speed limits (VSL), which consists in imposing variable location-dependent speed limits across the road network. A general overview of the theoretical background and the main strategies of variable speed limits frameworks is proposed by (Khondaker \& Kattan, 2015b).

Many VSL approaches can be found in the literature. Some of them aim at improving safety (Levin et al., 2019; Wu et al., 2019; Robinson et al., 2000), increasing road capacity and traffic flow (Frejo et al., 2019; Hadiuzzaman \& Qiu, 2013; Hegyi et al., 2008), reducing pollution (S. K. Zegeye et al., 2012), and improving the energy efficiency (Othman et al., 2020). Usually, all these approaches consist in harmonizing the traffic speed. Their control frameworks can therefore have a positive impact on several of these metrics (Du \& Razavi, 2019), and multi-criteria optimization may not always be necessary (Khondaker \& Kattan, 2015a). VSL strategies can be of particular interest in cases of poor visibility, especially under foggy conditions (Zhao et al., 2019).

Most VSL approaches for improved energy efficiency are designed to control a highway environment. For example, $\mathrm{Zu}$ et al. (2018) formulate a VSL problem as a convex quadratic optimization problem in order to minimize the global energy consumption. B. Liu et al. (2011) propose a VSL control strategy based on the vehicular trajectory that minimizes the fuel consumption of a single vehicle under certain traffic conditions. Another framework is proposed by S. K. Zegeye et al. (2012) to control VSL on highways. The authors implement an MPC strategy. Such approaches are valuable as MPC is compatible with the uncertainties of the traffic models, and it can handle non-linear and non-convex optimization. However, special attention must be paid to the computation time to make it tractable for real-time operation, especially when the control inputs are very numerous. To address this issue, MPC approaches can be parameterized to reduce the number of control inputs (Othman et al., 2020; S. K. Zegeye et al., 2012).

Some VSL approaches have been designed to be compliant in urban environments. For example, a framework to find the optimal speed limit of a single road section is proposed by De Nunzio et al. (2014). Usually, these strategies are based on multi-criteria objective functions in order to improve both the energy efficiency and the traffic performance, and avoid trivial solutions, e.g., minimum or maximum speed limits everywhere. Othman et al. (2020) propose to optimize the weighted sum of energy consumption and total distance traveled by road users, De Nunzio and Gutman (2017) minimize the energy consumption and the total time spent by vehicles by implementing a method based on shock waves theory, and Tajali and Hajbabaie (2018) harmonize the speed and maximize the outflow. Similarly, Van den Berg et al. (2007) propose a hybrid MPC framework aiming at minimizing the travel time in mixed urban and highway networks.

Machine learning strategies represent a promising opportunity for VSL implementation as they could greatly reduce computation times. For example, an RL approach aiming at optimizing the total network throughput, the delay time, and the emissions is proposed by F. Zhu and Ukkusuri (2014).

As mentioned previously, the efficiency of V2I communication-based VSL approaches can be enhanced when coordinated with cooperative strategies based on V2V and $\mathrm{I} 2 \mathrm{~V}$ communications. For instance, CACC systems can be subject to VSL control strategies for improved traffic fluidity and energy efficiency (Shladover et al., 2015).

\subsection{Traffic Light Adaptive Control}

In Section 2.4, we reviewed the use of $\mathrm{V} 2 \mathrm{~V}$ and/or $\mathrm{I} 2 \mathrm{~V}$ communication to control intersections, that can even be unsignalized when the penetration rate of CAVs reaches $100 \%$. In this section, we address the use of V2I communication in order to control the TLS, i.e. control their cycle time, split time and offset, and improve the overall energy efficiency of the vehicles (HVs and CAVs) traveling in the network. Note that instead of using V2I communication (for HVs that are not connected for example), one may imagine a system with fixed sensors in order to count and locate vehicles. These sensors include loop detectors, proximity sensors, or cameras associated with image classification systems (Kataria \& Rani, 2019). In this sense, many studies consider the control of TLS in order to maximize the bandwidth (Gomes, 2015) and minimize the travel time (Kamal et al., 2015; He et al., 2012). Several strategies aim at performing this function: SCOOT (Split, Cycle and Offset Optimisation Technique) (Hunt et al., 1981), SCATS (Sydney Coordinated Adaptive Traffic System) (Lowrie et al., 1982), RHODES (Real-time Hierarchical Optimized Distributed Effective System) (Mirchandani \& Head, 2001), TUC (Traffic-responsive Urban Control) (Dinopoulou et al., 2006), max-pressure (Varaiya, 2013), TRANSYT-7F, Synchro, PASSER, TSOP, etc. (Gomes, 2015). Also, few TLS control strategies explicitly consider the reduction of energy consumption (Osorio \& Nanduri, 2015; Khamis \& Gomaa, 2012; Stevanovic et al., 2009). 
On highways, it is possible to regulate the entering traffic flow through ramp metering, that can be applied on on-ramps. Some authors propose ramp metering approaches that aim at reducing traffic emissions (Csikós et al., 2011; Pasquale et al., 2015). An overview of ramp metering algorithms is proposed by Papageorgiou and Kotsialos (2002).

For optimal energy efficiency and improved throughput, both V2V/I2V and V2I communication-based approaches should be coordinated, i.e. a bi-directional CAV-infrastructure communication should be proposed (B. Xu et al., 2018; De Nunzio et al., 2017; Goodall et al., 2013). For example, B. Xu et al. (2018) present an approach that combines TLS control and eco-driving, i.e. the control of vehicles' speed profiles, in a $100 \%$ CAVs environment. The objective of this study is to optimize the TLS timing and the vehicles' arrival time at the intersection in order to reduce the total travel time and the overall energy consumption. The results reveal a significant improvement of energy efficiency and traffic performance, both under constant and varying traffic demand conditions. As mentioned in Section 2.4, the communication range has a significant impact on the performance of such approaches: a wider communication range gives better results. In this study, it seems that the better results are obtained with a communication range of about $800 \mathrm{~m}$. The TLS cycle length is also of major importance, and the ideal length is $60 \mathrm{~s}$, according to the authors. In the same vein, De Nunzio et al. (2017) present a strategy based on the same control actuators (traffic light offsets and recommended speeds) in order to maximize bandwidth along an arterial, which corresponds mainly to improved energy efficiency, lower idling time and number of stops. According to the authors, the impact on travel time mainly depends on the speed advisory. The results of this study show that this kind of approach outperforms other existing strategies, especially in comparison with TLS control and eco-driving when they are not simultaneously controlled. Also, the approach is particularly efficient under free-flow traffic conditions, and energy consumption is reduced without increasing travel time. TLS control can also be coordinated with CACC-enabled strategies. As indicated in Section 2.2, this could consist in arterial coordinated start, that coordinates the start of vehicles waiting at a red traffic signal when the signal turns green (Lioris et al., 2016; Shladover et al., 2015). To go further, one may also imagine a framework in which the TLS offsets are optimized in order to minimize the idling of CACC strings (H. Liu et al., 2019).

It is also possible to coordinate two control strategies that are both based on V2I communication. For example, De Nunzio et al. (2015) introduce a strategy that maximizes bandwidth along an arterial through TLS control and VSL. Here again, the objective is to improve the energy efficiency and reduce the travel time, and the authors indicate a potential dramatic reduction of energy consumption without increasing the travel time. Similarly, S. Liu et al. (2017) and S. K. Zegeye (2011) propose approaches that control both TLS and VSL to reduce energy consumption.

To improve the efficiency of TLS control strategies, the behavior of vehicles, especially HVs, should be considered and predicted. The coordination of multiple intersections, as in bandwidth maximization approaches, also opens the door to further improvement of both the energy efficiency and the traffic performance (B. Xu et al., 2018). Also, such systems need to be robust and adaptable to any type of intersection and network in order to be deployable on a large scale (De Nunzio et al., 2017). Finally, it would be interesting to analyze the system response to a traffic demand much higher than the network capacity (De Nunzio et al., 2015).

\section{Summary}

CAVs offer huge opportunities in terms of energy efficiency, pollutant emissions, throughput, safety, and comfort improvement because of the possibilities regarding the certainty of predictions of their behavior and the precision of their powertrain control. A first approach to reduce their energy consumption consists in the development of simple rule-based control strategies. However, these are usually not sufficient and optimization-based strategies appear to be necessary. Such strategies involve the need of precise and reliable energy consumption models, accurate traffic models for prediction purposes, performing optimization algorithms, as well as fast and longrange communication devices.

One first layer in the optimal control of CAVs corresponds to eco-routing and eco-driving strategies based on $\mathrm{V} 2 \mathrm{~V}$ and $\mathrm{I} 2 \mathrm{~V}$ communications. In this case, the vehicles are performing "individual gain" optimization. These approaches are more efficient when the CAVs penetration rate is high, because the predictions are more reliable and the need for guessing is reduced. Overall, the more knowledge a CAV has about its in-trip and final trip constraints before its departure, the more effective this kind of approach is. Some moot points remain regarding the consideration and the prediction of HVs' aggressiveness.

Another layer in the optimal control of CAVs is the development of cooperative strategies between CAVs in order to improve the overall energy efficiency. These approaches are also based on V2V and I2V communications, but vehicles are here considering the impact of their decisions on the behavior and the energy consumption of other vehicles, i.e. vehicles are performing a "common good" optimization. These control strategies include vehicle platooning, CACC, lane change and merge, and cooperative intersection control. For an optimal control, these cooperative strategies should perfectly integrate both vehicle-based radar data and GPS data transmitted through V2V communication. Also, CAVs can have a harmonizing effect on the speed of the surrounding traffic, especially in case of cooperating CAVs. This harmonization appears to have a beneficial impact on the global 
energy consumption, but it is difficult to estimate because it depends on the CAVs penetration rate, on traffic congestion, and on the network architecture.

Finally, one last layer consists in the control of infrastructures, especially TLS and VSL, which are based on V2I communication. These approaches are compatible with HVs, but the potential gains are greater with CAVs because they are more precise and have much shorter reaction times.

Based on the works reviewed in this chapter, the orders of magnitude of the gains in energy efficiency offered by the different control strategies are summarized in Table 2. When VSL and TLS approaches are combined, it appears that the energy consumption could be reduced by up to $40 \%$. It is essential to note that all the control strategies summarized in Table 2 are highly dependent on traffic conditions, CAVs penetration rate, network architecture, etc.

Table 2: Potential energy efficiency improvement of the control strategies presented in this chapter.

\begin{tabular}{|c|c|c|c|}
\hline \multicolumn{2}{|c|}{ Control strategy } & $\begin{array}{c}\text { Potential energy } \\
\text { efficiency improvement }\end{array}$ \\
\hline \multirow{3}{*}{ CAVs control } & \multirow{2}{*}{ Connected control } & Eco-routing & $10 \%-15 \%$ \\
\cline { 3 - 4 } & \multirow{2}{*}{ Cooperative control } & Eco-driving & $15 \%-40 \%$ \\
\cline { 3 - 4 } & & Car following & $20 \%$ \\
\cline { 3 - 4 } & & Harmonization effect & $20 \%-50 \%$ \\
\hline \multirow{2}{*}{ Infrastructure control } & \multicolumn{2}{|c|}{ Variable speed limits control } & $15 \%$ \\
\cline { 2 - 4 } & \multicolumn{2}{|c|}{ Traffic light signals control } & $15 \%$ \\
\hline
\end{tabular}

\section{Future Directions}

CAVs are considered the next major technological advance in the mobility sector. Not only do they have a crucial role to play in road safety, but they can also greatly improve traffic fluidity, as well as energy efficiency by reducing vehicle ownership and energy consumption rate (F. Liu et al., 2019). However, at system-wide level, the effect of CAVs on traffic congestion and energy efficiency is still uncertain and these technologies might increase the total fuel consumption (Brown et al., 2014; U.S. Department of Energy, 2018).

In order to optimize the overall energy efficiency, it seems that coordinating complementary control strategies is promising. In the next years, Additional approaches involving CAVs are probably yet to be found, because of the large field of possibilities offered by these technologies. Some directions already investigated in the literature include:

- cooperative intersection control and CACC (Jin et al., 2013);

- VSL and CACC (Shladover et al., 2015);

- TLS control and eco-driving (B. Xu et al., 2018; De Nunzio et al., 2017);

- TLS control and VSL De Nunzio et al. (2015).

To ensure the efficiency of such approaches, as well as the safety of road users and pedestrians, it is essential to establish efficient and robust communications under highly dynamic environments, and to consider the uncertainty over the bounds on the predicted vehicle speed. These control approaches should be robust for all types of powertrains, all types of road network and intersection, and under all congestion conditions. In fact, such systems currently lack of ability to address diverse real-world driving conditions (Dey et al., 2015). Also, the increasing number of connected vehicles on the roads leads to an increasing amount of available data, which requires better computing capacities. In this context, machine learning methods seem to be a promising trend both for prediction and control purposes, as they are adaptable to all types of control actuators and strategies.

In the future, additional issues need to be addressed to democratize the circulation of CAVs on a large scale. These includes, inter alia, the legal aspects of crashes caused by failure of the system, users' privacy and security, technology certification, and user training in the case of vehicles that are not fully autonomous. One moot point in the public debate is the resistance of such systems to cyber attacks.

Finally, the democratization of CAVs on a large scale requires convincing the public authorities and/or the vehicles manufacturers to invest or change current policies to promote these technologies. To do this, a real standardized simulation platform with representative scenarios that clearly show the benefits of deploying CAVs on the roads seems to be missing. This is almost essential as real-world experiments in traffic control are very long and expensive to put in place. 


\section{References}

Aarts, L., \& Feddes, G. (2016). European truck platooning challenge. In International symposium on heavy vehicle transport technology.

Ahmane, M., Abbas-Turki, A., Perronnet, F., Wu, J., El Moudni, A., Buisson, J., \& Zeo, R. (2013). Modeling and controlling an isolated urban intersection based on cooperative vehicles. Transportation Research Part C: Emerging Technologies, 28, 44-62.

Al Alam, A., Gattami, A., \& Johansson, K. H. (2010). An experimental study on the fuel reduction potential of heavy duty vehicle platooning. In 13th international ieee conference on intelligent transportation systems (pp. 306-311).

An, H., \& Jung, J.-i. (2018). Design of a cooperative lane change protocol for a connected and automated vehicle based on an estimation of the communication delay. Sensors, 18(10), 3499.

Andersen, H., Shen, X., Eng, Y. H., Rus, D., \& Ang Jr, M. H. (2017). Connected cooperative control of autonomous vehicles during unexpected road situations. Mechanical Engineering, 139(12), S3-S7.

Asadi, B., Zhang, C., \& Vahidi, A. (2010). The role of traffic flow preview for planning fuel optimal vehicle velocity. In Dynamic systems and control conference (Vol. 44182, pp. 813-819).

Awal, T., Murshed, M., \& Ali, M. (2015). An efficient cooperative lane-changing algorithm for sensorand communication-enabled automated vehicles. In 2015 ieee intelligent vehicles symposium (iv) (pp. 1328-1333).

Bhoopalam, A. K., Agatz, N., \& Zuidwijk, R. (2018). Planning of truck platoons: A literature review and directions for future research. Transportation Research Part B: Methodological, 107, 212-228.

Bodenheimer, R., Brauer, A., Eckhoff, D., \& German, R. (2014). Enabling glosa for adaptive traffic lights. In 2014 ieee vehicular networking conference (vnc) (pp. 167-174).

Bonnet, C., \& Fritz, H. (2000). Fuel consumption reduction in a platoon: Experimental results with two electronically coupled trucks at close spacing (Tech. Rep.). SAE Technical Paper.

Browand, F., McArthur, J., \& Radovich, C. (2004). Fuel saving achieved in the field test of two tandem trucks. UC Berkeley: California Partners for Advanced Transportation Technology.

Brown, A., Gonder, J., \& Repac, B. (2014). An analysis of possible energy impacts of automated vehicles. In G. Meyer \& S. Beiker (Eds.), Road vehicle automation (pp. 137-153). Cham: Springer.

Charalampidis, A. C., \& Gillet, D. (2014). Speed profile optimization for vehicles crossing an intersection under a safety constraint. In 2014 european control conference (ecc) (pp. 2894-2901).

Continental. (2019). Continental launches smart city mobility and transportation hub for safer and smarter cities. https://www.continental.com/en-us/press-/press-releases/smart-city -mobility-205048.

Csikós, A., Luspay, T., \& Varga, I. (2011). Modeling and optimal control of travel times and traffic emission on freeways. IFAC Proceedings Volumes, 44(1), 13058 - 13063. (18th IFAC World Congress)

De Nunzio, G., Gomes, G., Canudas-de-Wit, C., Horowitz, R., \& Moulin, P. (2017). Speed advisory and signal offsets control for arterial bandwidth maximization and energy consumption reduction. IEEE Transactions on Control Systems Technology, 25(3), 875-887.

De Nunzio, G., Sciarretta, A., Ben Gharbia, I., \& Ojeda, L. L. (2018). A constrained eco-routing strategy for hybrid electric vehicles based on semi-analytical energy management. In 2018 21st international conference on intelligent transportation systems (itsc) (p. 355-361).

De Nunzio, G., \& Thibault, L. (2017). Energy-optimal driving range prediction for electric vehicles. In 2017 ieee intelligent vehicles symposium (iv) (p. 1608-1613).

De Nunzio, G., Canudas-de-Wit, C., \& Moulin, P. (2014). Urban traffic eco-driving: A macroscopic steady-state analysis. In Control conference (ecc), 2014 european (pp. 2581-2587).

De Nunzio, G., Canudas-de-Wit, C., Moulin, P., \& Di Domenico, D. (2016). Eco-driving in urban traffic networks using traffic signals information. International Journal of Robust and Nonlinear Control, $26(6), 1307-1324$.

De Nunzio, G., Gomes, G., Canudas-de-Wit, C., Horowitz, R., \& Moulin, P. (2015). Arterial bandwidth maximization via signal offsets and variable speed limits control. In Decision and control (cdc), 2015 ieee 54th annual conference on (pp. 5142-5148).

De Nunzio, G., \& Gutman, P.-O. (2017). An application of shock wave theory to urban traffic control via dynamic speed advisory. In heart 2017: 6th symposium of the european association for research in transportation.

De Nunzio, G., Thibault, L., \& Sciarretta, A. (2017a). Bi-objective eco-routing in large urban road networks. In 2017 ieee 20th international conference on intelligent transportation systems (itsc) 
(p. 1-7)

De Nunzio, G., Thibault, L., \& Sciarretta, A. (2017b). Model-based eco-routing strategy for electric vehicles in large urban networks. In Comprehensive energy management-eco routing \& velocity profiles (pp. 81-99). Springer.

Dey, K. C., Yan, L., Wang, X., Wang, Y., Shen, H., Chowdhury, M., .. Soundararaj, V. (2015). A review of communication, driver characteristics, and controls aspects of cooperative adaptive cruise control (cacc). IEEE Transactions on Intelligent Transportation Systems, 17(2), 491-509.

Dinopoulou, V., Diakaki, C., \& Papageorgiou, M. (2006). Applications of the urban traffic control strategy tuc. European Journal of Operational Research, $175(3), 1652$ - 1665.

Di Vaio, M., Fiengo, G., Petrillo, A., Salvi, A., Santini, S., \& Tufo, M. (2019). Cooperative shock waves mitigation in mixed traffic flow environment. IEEE Transactions on Intelligent Transportation Systems, 20(12), 4339-4353.

Dollar, A., Sciarretta, A., \& Vahidi, A. (2020a). Information and collaboration levels in vehicular strings: A comparative study. IFAC Proceedings Volumes.

Dollar, A., Sciarretta, A., \& Vahidi, A. (2020b). Multi-agent control of lane-switching automated vehicles for energy efficiency. In 2020 american control conference (acc) (pp. 422-429).

Du, S., \& Razavi, S. (2019). Variable speed limit for freeway work zone with capacity drop using discrete-time sliding mode control. Journal of Computing in Civil Engineering, 33(2), 04019001.

Ericsson, E., Larsson, H., \& Brundell-Freij, K. (2006). Optimizing route choice for lowest fuel consumption-potential effects of a new driver support tool. Transportation Research Part C: Emerging Technologies, 14(6), 369-383.

Fayazi, S. A., \& Vahidi, A. (2018). Mixed-integer linear programming for optimal scheduling of autonomous vehicle intersection crossing. IEEE Transactions on Intelligent Vehicles, 3(3), 287-299.

Fayazi, S. A., Vahidi, A., \& Luckow, A. (2017). Optimal scheduling of autonomous vehicle arrivals at intelligent intersections via milp. In 2017 american control conference (acc) (pp. 4920-4925).

Fontaras, G., Zacharof, N.-G., \& Ciuffo, B. (2017). Fuel consumption and co2 emissions from passenger cars in europe-laboratory versus real-world emissions. Progress in Energy and Combustion Science, 60, 97-131.

Frejo, J. R. D., Papamichail, I., Papageorgiou, M., \& De Schutter, B. (2019). Macroscopic modeling of variable speed limits on freeways. Transportation research part C: emerging technologies, 100, $15-33$.

Ghiasi, A., Li, X., \& Ma, J. (2019). A mixed traffic speed harmonization model with connected autonomous vehicles. Transportation Research Part C: Emerging Technologies, 104, 210-233.

Giammarino, V., Baldi, S., Frasca, P., \& Monache, M. L. D. (2020). Traffic flow on a ring with a single autonomous vehicle: An interconnected stability perspective. IEEE Transactions on Intelligent Transportation Systems, 1-11.

Gomes, G. (2015). Bandwidth maximization using vehicle arrival functions. IEEE Transactions on Intelligent Transportation Systems, 16(4), 1977-1988.

Goodall, N. J., Smith, B. L., \& Park, B. (2013). Traffic signal control with connected vehicles. Transportation Research Record, 2381(1), 65-72.

Guanetti, J., Kim, Y., \& Borrelli, F. (2018). Control of connected and automated vehicles: State of the art and future challenges. Annual Reviews in Control, 45, 18-40.

Hadiuzzaman, M., \& Qiu, T. Z. (2013). Cell transmission model based variable speed limit control for freeways. Canadian Journal of Civil Engineering, 40(1), 46-56.

Han, J., Sciarretta, A., Ojeda, L. L., De Nunzio, G., \& Thibault, L. (2018). Safe-and eco-driving control for connected and automated electric vehicles using analytical state-constrained optimal solution. IEEE Transactions on Intelligent Vehicles, 3(2), 163-172.

Han, J., Vahidi, A., \& Sciarretta, A. (2019). Fundamentals of energy efficient driving for combustion engine and electric vehicles: An optimal control perspective. Automatica, 103, 558 - 572.

He, Q., Head, K. L., \& Ding, J. (2012). Pamscod: Platoon-based arterial multi-modal signal control with online data. Transportation Research Part C: Emerging Technologies, 20(1), 164-184.

Hegyi, A., Hoogendoorn, S. P., Schreuder, M., Stoelhorst, H., \& Viti, F. (2008). Specialist: A dynamic speed limit control algorithm based on shock wave theory. In 2008 11th international ieee conference on intelligent transportation systems (p. 827-832).

Heß, D., Lattarulo, R., Pérez, J., Schindler, J., Hesse, T., \& Köster, F. (2018). Fast maneuver planning for cooperative automated vehicles. In 2018 21st international conference on intelligent transportation systems (itsc) (pp. 1625-1632). 
Huang, S., Sadek, A. W., \& Zhao, Y. (2012). Assessing the mobility and environmental benefits of reservation-based intelligent intersections using an integrated simulator. IEEE Transactions on Intelligent Transportation Systems, 13(3), 1201-1214.

Huang, Y., Ng, E. C., Zhou, J. L., Surawski, N. C., Chan, E. F., \& Hong, G. (2018). Eco-driving technology for sustainable road transport: A review. Renewable and Sustainable Energy Reviews, 93, 596-609.

Hunt, P., Robertson, D., Bretherton, R., \& Winton, R. (1981). Scoot-a traffic responsive method of coordinating signals (Tech. Rep.).

IEA. (2017a). Energy consumption in transport in selected iea countries. https://www.iea.org/ data-and-statistics/charts/energy-consumption-in-transport-in-selected-iea -countries-2017.

IEA. (2017b). Largest end-uses of energy by sector in selected iea countries. https://www.iea.org/ data-and-statistics/charts/largest-end-uses-of-energy-by-sector-in-selected-iea -countries-2017.

Jin, Q., Wu, G., Boriboonsomsin, K., \& Barth, M. (2013). Platoon-based multi-agent intersection management for connected vehicle. In 16th international ieee conference on intelligent transportation systems (itsc 2013) (pp. 1462-1467).

Jin, Q., Wu, G., Boriboonsomsin, K., \& Barth, M. J. (2016). Power-based optimal longitudinal control for a connected eco-driving system. IEEE Transactions on Intelligent Transportation Systems, $17(10), 2900-2910$.

Kamal, M. A. S., Imura, J.-i., Hayakawa, T., Ohata, A., \& Aihara, K. (2015). Traffic signal control of a road network using milp in the mpc framework. International journal of intelligent transportation systems research, 13(2), 107-118.

Kamal, M. A. S., Taguchi, S., \& Yoshimura, T. (2016). Efficient driving on multilane roads under a connected vehicle environment. IEEE Transactions on Intelligent Transportation Systems, 17(9), 2541-2551.

Kataria, P., \& Rani, A. (2019). Real-time traffic light management system with manual control. In 2019 3rd international conference on recent developments in control, automation \& power engineering (rdcape) (pp. 419-424).

Khamis, M. A., \& Gomaa, W. (2012). Enhanced multiagent multi-objective reinforcement learning for urban traffic light control. In 2012 11th international conference on machine learning and applications (Vol. 1, pp. 586-591).

Khondaker, B., \& Kattan, L. (2015a). Variable speed limit: a microscopic analysis in a connected vehicle environment. Transportation Research Part C: Emerging Technologies, 58, 146 - 159.

Khondaker, B., \& Kattan, L. (2015b). Variable speed limit: an overview. Transportation Letters, 7(5), $264-278$.

Kluge, S., Santa, C., Dangl, S., Wild, S., Brokate, M., Reif, K., \& Busch, F. (2013). On the computation of the energy-optimal route dependent on the traffic load in ingolstadt. Transportation Research Part C: Emerging Technologies, 36, 97 - 115.

Kubička, M., Klusáček, J., Sciarretta, A., Cela, A., Mounier, H., Thibault, L., \& Niculescu, S.-I. (2016). Performance of current eco-routing methods. In Intelligent vehicles symposium (iv), 2016 ieee (pp. 472-477).

Kuo, Y. (2010). Using simulated annealing to minimize fuel consumption for the time-dependent vehicle routing problem. Computers \& Industrial Engineering, 59(1), 157-165.

Larson, J., Liang, K.-Y., \& Johansson, K. H. (2014). A distributed framework for coordinated heavy-duty vehicle platooning. IEEE Transactions on Intelligent Transportation Systems, 16(1), 419-429.

Lee, J., Kim, J., Park, J., \& Bae, C. (2013). Effect of the air-conditioning system on the fuel economy in a gasoline engine vehicle. Proceedings of the Institution of Mechanical Engineers, Part D: Journal of Automobile Engineering, 227(1), 66-77.

Levin, M., Chen, R., Liao, C.-F., \& Zhang, T. (2019). Improving intersection safety through variable speed limits for connected vehicles (no. cts 19-12) (Tech. Rep.). Roadway Safety Institute.

Li, X., \& Sun, J.-Q. (2017). Studies of vehicle lane-changing dynamics and its effect on traffic efficiency, safety and environmental impact. Physica A: Statistical Mechanics and its Applications, 467, 41 58.

Liang, K.-Y., Mårtensson, J., \& Johansson, K. H. (2013). When is it fuel efficient for a heavy duty vehicle to catch up with a platoon? IFAC Proceedings Volumes, 46(21), 738-743.

Liard, T., Stern, R., \& Delle Monache, M.-L. (2020, July). Optimal driving strategies for traffic control with autonomous vehicles. In The 21rst IFAC World Congress. 
Lin, D., \& Jabari, S. E. (2020). Pay for intersection priority: A free market mechanism for connected vehicles. arXiv preprint arXiv:2001.01813.

Lin, D., Li, L., \& Jabari, S. E. (2019). Pay to change lanes: A cooperative lane-changing strategy for connected/automated driving. Transportation Research Part C: Emerging Technologies, 105, 550-564.

Lioris, J., Pedarsani, R., Tascikaraoglu, F. Y., \& Varaiya, P. (2016). Doubling throughput in urban roads by platooning. IFAC-PapersOnLine, 49(3), 49-54.

Litman, T. (2017). Autonomous vehicle implementation predictions. Victoria Transport Policy Institute Victoria, Canada.

Liu, B., Ghosal, D., Chuah, C.-N., \& Zhang, H. M. (2011). Reducing greenhouse effects via fuel consumption-aware variable speed limit (fc-vsl). IEEE Transactions on Vehicular Technology, 61(1), 111-122.

Liu, F., Zhao, F., Liu, Z., \& Hao, H. (2019). Can autonomous vehicle reduce greenhouse gas emissions? a country-level evaluation. Energy Policy, 132, 462 - 473.

Liu, H., Lu, X.-Y., \& Shladover, S. E. (2019). Traffic signal control by leveraging cooperative adaptive cruise control (cacc) vehicle platooning capabilities. Transportation research part C: emerging technologies, 104, 390-407.

Liu, H., Shladover, S. E., Lu, X.-Y., \& Kan, X. (2020). Freeway vehicle fuel efficiency improvement via cooperative adaptive cruise control. Journal of Intelligent Transportation Systems, 1-13.

Liu, P., Ozguner, U., \& Zhang, Y. (2017). Distributed mpc for cooperative highway driving and energy-economy validation via microscopic simulations. Transportation Research Part C: Emerging Technologies, 77, 80-95.

Liu, S., Hellendoorn, H., \& De Schutter, B. (2017). Model predictive control for freeway networks based on multi-class traffic flow and emission models. IEEE Transactions on Intelligent Transportation Systems, $18(2), 306-320$.

Lombard, A., Perronnet, F., Abbas-Turki, A., \& El Moudni, A. (2017). On the cooperative automatic lane change: Speed synchronization and automatic "courtesy". In Design, automation \& test in europe conference $\&$ exhibition (date), 2017 (pp. 1655-1658).

Lowrie, P., et al. (1982). The sydney co-ordinated adaptive traffic system (scats) - principles, methodology, algorithms. In Proceedings of the international conference on road traffic signaling, london, $u k$.

Lu, X.-Y., \& Shladover, S. E. (2011). Automated truck platoon control (Tech. Rep.). Institute of Transportation Studies, UC Berkeley.

Marcu, B., \& Browand, F. (1999). Aerodynamic forces experienced by a 3-vehicle platoon in a crosswind (Tech. Rep.). SAE Technical Paper.

McAuliffe, B., Croken, M., Ahmadi-Baloutaki, M., \& Raeesi, A. (2017). Fuel-economy testing of a three-vehicle truck platooning system. UC Berkeley.

Miculescu, D., \& Karaman, S. (2019). Polling-systems-based autonomous vehicle coordination in traffic intersections with no traffic signals. IEEE Transactions on Automatic Control, 65(2), 680-694.

Milanés, V., Shladover, S. E., Spring, J., Nowakowski, C., Kawazoe, H., \& Nakamura, M. (2013). Cooperative adaptive cruise control in real traffic situations. IEEE Transactions on intelligent transportation systems, $15(1), 296-305$.

Mirchandani, P., \& Head, L. (2001). A real-time traffic signal control system: architecture, algorithms, and analysis. Transportation Research Part C: Emerging Technologies, 9(6), 415 - 432.

Namazi, E., Li, J., \& Lu, C. (2019). Intelligent intersection management systems considering autonomous vehicles: a systematic literature review. IEEE Access, 7, 91946-91965.

Nannicini, G., Delling, D., Schultes, D., \& Liberti, L. (2012). Bidirectional a* search on time-dependent road networks. Networks, 59(2), 240-251.

Nie, J., Zhang, J., Ding, W., Wan, X., Chen, X., \& Ran, B. (2016). Decentralized cooperative lanechanging decision-making for connected autonomous vehicles. IEEE Access, 4, 9413-9420.

Noorvand, H., Karnati, G., \& Underwood, B. S. (2017). Autonomous vehicles: assessment of the implications of truck positioning on flexible pavement performance and design. Transportation Research Record, 2640(1), 21-28.

Nowakowski, C., O'Connell, J., Shladover, S. E., \& Cody, D. (2010). Cooperative adaptive cruise control: Driver acceptance of following gap settings less than one second. In Proceedings of the human factors and ergonomics society annual meeting (Vol. 54, pp. 2033-2037).

Ojeda, L. L., Chasse, A., \& Goussault, R. (2017). Fuel consumption prediction for heavy-duty vehicles using digital maps. In 2017 ieee 20th international conference on intelligent transportation systems 
(itsc) (p. 1-7).

Ojeda, L. L., Han, J., Sciarretta, A., De Nunzio, G., \& Thibault, L. (2017). A real-time eco-driving strategy for automated electric vehicles. In 2017 ieee 56 th annual conference on decision and control (cdc) (p. 2768-2774).

Osorio, C., \& Nanduri, K. (2015). Energy-efficient urban traffic management: a microscopic simulationbased approach. Transportation Science, 49(3), 637-651.

Othman, B., De Nunzio, G., Di Domenico, D., \& Canudas-de-Wit, C. (2019). Ecological traffic management: A review of the modeling and control strategies for improving environmental sustainability of road transportation. Annual Reviews in Control, 48, 292 - 311.

Othman, B., De Nunzio, G., Di Domenico, D., \& Canudas-de-Wit, C. (2020). Variable speed limits control in an urban road network to reduce environmental impact of traffic. In 2020 annual american control conference (acc) (pp. 1179-1184).

Papageorgiou, M., \& Kotsialos, A. (2002). Freeway ramp metering: An overview. IEEE transactions on intelligent transportation systems, 3(4), 271-281.

Pasquale, C., Papamichail, I., Roncoli, C., Sacone, S., Siri, S., \& Papageorgiou, M. (2015). Twoclass freeway traffic regulation to reduce congestion and emissions via nonlinear optimal control. Transportation Research Part C: Emerging Technologies, 55, 85-99.

Peloton technology. (n.d.). https://peloton-tech.com/how-it-works. (Accessed: 2020-08-14)

Pinto, G., \& Oliver-Hoyo, M. T. (2008). Using the relationship between vehicle fuel consumption and co2 emissions to illustrate chemical principles. Journal of Chemical Education, 85(2), 218.

Pulkrabek, W. W. (2004). Engineering Fundamentals of the Internal Combustion Engine, 2nd Ed. Journal of Engineering for Gas Turbines and Power, 126(1), 198-198.

Qi, Y. G., Teng, H. H., \& Yu, L. (2004). Microscale emission models incorporating acceleration and deceleration. Journal of Transportation Engineering, 130(3), 348-359.

Qom, S. F., Xiao, Y., \& Hadi, M. (2016). Evaluation of cooperative adaptive cruise control (cacc) vehicles on managed lanes utilizing macroscopic and mesoscopic simulation. In Transportation research board 95th annual meeting.

Raboy, K., Ma, J., Leslie, E., \& Zhou, F. (2020). A proof-of-concept field experiment on cooperative lane change maneuvers using a prototype connected automated vehicle testing platform. Journal of Intelligent Transportation Systems, 1-16.

Raboy, K., Ma, J., Stark, J., Zhou, F., Rush, K., \& Leslie, E. (2017). Cooperative control for lane change maneuvers with connected automated vehicles: A field experiment (Tech. Rep.).

Robinson, M., et al. (2000). Examples of variable speed limit applications.

Sciarretta, A., Nunzio, G. D., \& Ojeda, L. L. (2015). Optimal ecodriving control: Energy-efficient driving of road vehicles as an optimal control problem. IEEE Control Systems Magazine, 35(5), 71-90.

Sciarretta, A., \& Vahidi, A. (2020a). Energy saving potentials of cavs. In Energy-efficient driving of road vehicles: Toward cooperative, connected, and automated mobility (pp. 1-31). Cham: Springer International Publishing.

Sciarretta, A., \& Vahidi, A. (2020b). Fundamentals of vehicle modeling. In Energy-efficient driving of road vehicles: Toward cooperative, connected, and automated mobility (pp. 33-62). Cham: Springer International Publishing.

Shladover, S. E., Nowakowski, C., Lu, X.-Y., \& Ferlis, R. (2015). Cooperative adaptive cruise control: Definitions and operating concepts. Transportation Research Record, 2489(1), 145-152.

Stern, R. E., Chen, Y., Churchill, M., Wu, F., Monache, M. L. D., Piccoli, B., .. Work, D. B. (2019). Quantifying air quality benefits resulting from few autonomous vehicles stabilizing traffic. Transportation Research Part D: Transport and Environment, 67, 351 - 365.

Stevanovic, A., Stevanovic, J., Zhang, K., \& Batterman, S. (2009). Optimizing traffic control to reduce fuel consumption and vehicular emissions: Integrated approach with vissim, cmem, and visgaost. Transportation Research Record, 2128(1), 105-113.

Tachet, R., Santi, P., Sobolevsky, S., Reyes-Castro, L. I., Frazzoli, E., Helbing, D., \& Ratti, C. (2016). Revisiting street intersections using slot-based systems. PloS one, $11(3)$, e0149607.

Tajali, M., \& Hajbabaie, A. (2018, 03). Dynamic speed harmonization in urban street networks. Computer-Aided Civil and Infrastructure Engineering.

Talavera, E., Díaz-Álvarez, A., Jiménez, F., \& Naranjo, J. E. (2018). Impact on congestion and fuel consumption of a cooperative adaptive cruise control system with lane-level position estimation. Energies, $11(1), 194$.

Thibault, L., Nunzio, G. D., \& Sciarretta, A. (2018). A unified approach for electric vehicles range 
maximization via eco-routing, eco-driving, and energy consumption prediction. IEEE Transactions on Intelligent Vehicles, 3, 463-475.

U.S. Department of Energy. (2018). Autonomous vehicles: Uncertainties and energy implications.

U.S. Energy Information Administration. (2017). International energy outlook.

Van Arem, B., Van Driel, C. J., \& Visser, R. (2006). The impact of cooperative adaptive cruise control on traffic-flow characteristics. IEEE Transactions on intelligent transportation systems, 7(4), 429436.

Van de Hoef, S. (2016). Fuel-efficient centralized coordination of truck platooning (Unpublished doctoral dissertation). KTH Royal Institute of Technology.

Van De Hoef, S., Johansson, K. H., \& Dimarogonas, D. V. (2015). Fuel-optimal centralized coordination of truck platooning based on shortest paths. In 2015 american control conference (acc) (pp. 37403745).

Van den Berg, M., Hegyi, A., De Schutter, B., \& Hellendoorn, H. (2007). Integrated traffic control for mixed urban and freeway networks: A model predictive control approach. European Journal of Transport and Infrastructure Research EJTIR, 7 (3).

Varaiya, P. (2013). The max-pressure controller for arbitrary networks of signalized intersections. In Advances in dynamic network modeling in complex transportation systems (pp. 27-66). Springer.

Vinitsky, E., Kreidieh, A., Le Flem, L., Kheterpal, N., Jang, K., Wu, C., .. Bayen, A. M. (2018). Benchmarks for reinforcement learning in mixed-autonomy traffic. In Conference on robot learning (pp. 399-409).

Wadud, Z., MacKenzie, D., \& Leiby, P. (2016). Help or hindrance? the travel, energy and carbon impacts of highly automated vehicles. Transportation Research Part A: Policy and Practice, 86, $1-18$.

Wan, N., Vahidi, A., \& Luckow, A. (2016). Optimal speed advisory for connected vehicles in arterial roads and the impact on mixed traffic. Transportation Research Part C: Emerging Technologies, $69,548-563$.

Wan, N., Zhang, C., \& Vahidi, A. (2017). Probabilistic anticipation and control in autonomous car following. IEEE Transactions on Control Systems Technology, 27(1), 30-38.

Wang, M., van Maarseveen, S., Happee, R., Tool, O., \& van Arem, B. (2019). Benefits and risks of truck platooning on freeway operations near entrance ramp. Transportation Research Record, 2673(8), $588-602$.

Wang, Z., Wu, G., Hao, P., Boriboonsomsin, K., \& Barth, M. (2017). Developing a platoon-wide ecocooperative adaptive cruise control (cacc) system. In 2017 ieee intelligent vehicles symposium (iv) (pp. 1256-1261).

Wu, Y., Abdel-Aty, M., Wang, L., \& Rahman, M. S. (2019). Improving flow and safety in low visibility conditions by applying connected vehicles and variable speed limits technologies (Tech. Rep.).

Xia, H., Boriboonsomsin, K., \& Barth, M. (2013). Dynamic eco-driving for signalized arterial corridors and its indirect network-wide energy/emissions benefits. Journal of Intelligent Transportation Systems, 17(1), 31-41.

Xu, B., Ban, X. J., Bian, Y., Li, W., Wang, J., Li, S. E., \& Li, K. (2018). Cooperative method of traffic signal optimization and speed control of connected vehicles at isolated intersections. IEEE Transactions on Intelligent Transportation Systems, 20(4), 1390-1403.

Xu, Z., Kang, Y., \& Lv, W. (2017). Analysis and prediction of vehicle exhaust emission using ann. In 2017 36th chinese control conference (ccc) (pp. 4029-4033).

Yang, L., Hu, X., \& Fang, L. (2018). Carbon emissions tax policy of urban road traffic and its application in panjin, china. PloS one, $13(5)$.

Zegeye, S., De Schutter, B., Hellendoorn, J., Breunesse, E., \& Hegyi, A. (2013). Integrated macroscopic traffic flow, emission, and fuel consumption model for control purposes. Transportation Research Part C: Emerging Technologies, 31, 158-171.

Zegeye, S. K. (2011). Model-based traffic control for sustainable mobility.

Zegeye, S. K., De Schutter, B., Hellendoorn, J., Breunesse, E. A., \& Hegyi, A. (2012). A predictive traffic controller for sustainable mobility using parameterized control policies. IEEE Transactions on Intelligent Transportation Systems, 13(3), 1420-1429.

Zeng, W., Miwa, T., \& Morikawa, T. (2016). Prediction of vehicle co2 emission and its application to eco-routing navigation. Transportation Research Part C: Emerging Technologies, 68, 194-214.

Zhang, L., Chen, F., Ma, X., \& Pan, X. (2020). Fuel economy in truck platooning: A literature overview and directions for future research. Journal of Advanced Transportation, 2020.

Zhang, Y., \& Cassandras, C. G. (2018). The penetration effect of connected automated vehicles in urban 
traffic: an energy impact study. In 2018 ieee conference on control technology and applications (ccta) (pp. 620-625).

Zhao, X., Xu, W., Ma, J., Li, H., Chen, Y., \& Rong, J. (2019). Effects of connected vehicle-based variable speed limit under different foggy conditions based on simulated driving. Accident Analysis Es Prevention, 128, 206-216.

Zhu, F., \& Ukkusuri, S. V. (2014). Accounting for dynamic speed limit control in a stochastic traffic environment: A reinforcement learning approach. Transportation Research Part C: Emerging Technologies, 41, 30 - 47.

Zhu, J., Ngo, C., \& Sciarretta, A. (2019). Real-time optimal eco-driving for hybrid-electric vehicles. IFAC-PapersOnLine, 52(5), 562 - 567. (9th IFAC Symposium on Advances in Automotive Control AAC 2019)

Zu, Y., Liu, C., Dai, R., Sharma, A., \& Dong, J. (2018). Real-time energy-efficient traffic control via convex optimization. Transportation Research Part C: Emerging Technologies, 92, 119 - 136. 